\title{
Aldehyde-Selective Wacker Oxidation in a Thiyl-Mediated Vinyl Group Transfer Route to Daunosamine
}

\author{
Gregory K. Friestad, * Tao Jiang, and Alex K. Mathies \\ Department of Chemistry, University of Iowa, Iowa City, Iowa, 52242 \\ gregory-friestad@uiowa.edu
}

\section{Supporting Information}

\begin{abstract}
Contents
Procedures and Characterization Data for New Compounds

pp. $1-5$

${ }^{1} \mathrm{H}$ and ${ }^{13} \mathrm{C}$ NMR Spectra for New Compounds.

pp. 6-19

Comparison of Selected Daunosamine Syntheses

p. 20
\end{abstract}

Materials and Methods. Reactions employed oven- or flame-dried glassware under nitrogen unless otherwise noted. THF, diethyl ether, benzene and toluene were distilled from sodium/benzophenone ketyl under argon. $\mathrm{CH}_{2} \mathrm{Cl}_{2}$ was distilled from $\mathrm{CaH}_{2}$ under argon or nitrogen. Alternatively, these solvents were purchased inhibitor-free and were sparged with argon and passed through columns of activated alumina prior to use (dropwise addition of blue benzophenone ketyl solution revealed the THF purified in this manner sustained the blue color more readily than the control sample purified by distillation). Nitrogen was passed successively through columns of anhydrous $\mathrm{CaSO}_{4}$ and R3-11 catalyst for removal of water and oxygen, respectively. All other materials were used as received from commercial sources unless otherwise noted. Thin layer chromatography (TLC) employed glass $0.25 \mathrm{~mm}$ silica gel plates with UV indicator. Flash chromatography columns were packed with 230-400 mesh silica gel as a slurry in the initial elution solvent. Gradient flash chromatography was conducted by adsorption of product mixtures on silica gel, packing over a short pad of clean silica gel as a slurry in hexane, and eluting with a continuous gradient from hexane to the indicated solvent. Radial chromatography refers to centrifugally accelerated thin-layer chromatography performed with a Chromatotron using commercially supplied rotors. Melting points are uncorrected. Nuclear magnetic resonance (NMR) data were obtained at operating frequencies of 500 or $300 \mathrm{MHz}$ for ${ }^{1} \mathrm{H}$ and 125 or $75 \mathrm{MHz}$ for ${ }^{13} \mathrm{C}$. Infrared spectra were recorded using a single beam FT-IR spectrophotometer by standard transmission methods or by use of an attenuated total reflectance (ATR) probe. Optical rotations were determined using a digital polarimeter operating at ambient temperature. Low resolution mass spectra were obtained using sample introduction by dip, liquid chromatography or gas chromatography. High resolution mass spectra and combustion analyses were obtained from external commercial and institutional services. Chromatographic diastereomer ratio analyses employed GCMS with $15 \mathrm{~mL}$ x $0.25 \mathrm{~mm}$ I.D X $0.25 \mu$ F.T $5 \%$-phenyl-95\%-dimethylsiloxane column and helium as mobile phase or HPLC with Microsorb-MV Si 8um 100A or Chiralcel OD columns (2-propanol/hexane as mobile phase) or Chirex 3014 column (chloroform/hexane as mobile phase). 
(E)-1,1-dibenzyl-2-(but-2-enylidene)hydrazine (2). To a solution of (E)crotonaldehyde $(0.58 \mathrm{~mL}, 7.05 \mathrm{mmol})$ in toluene $(5 \mathrm{~mL})$ was added dibenzylhydrazine $(1.0 \mathrm{~g}, 4.7 \mathrm{mmol})$ and the mixture was stirred at room temperature for $16 \mathrm{~h}$. Concentration and flash chromatography (10:1 petroleum ether/ethyl acetate) afforded 2 (1.22 g, 98\%) as a pale yellow oil. (E)-Isomer: IR (film, $\mathrm{CHCl}_{3}$ ) 3028, 1453, 1133, $970 \mathrm{~cm}^{-1}$; ${ }^{1} \mathrm{H} \mathrm{NMR}(500 \mathrm{MHz}$, $\left.\mathrm{CDCl}_{3}\right) \delta 7.36-7.22(\mathrm{~m}, 10 \mathrm{H}), 6.95(\mathrm{~d}, J=8.8 \mathrm{~Hz}, 1 \mathrm{H}), 6.22(\mathrm{ddq}, J=15.5,8.9,1.7 \mathrm{~Hz}, 1 \mathrm{H}), 5.62(\mathrm{dq}, J$ $=15.5,6.8 \mathrm{~Hz}, 1 \mathrm{H}), 4.39(\mathrm{~s}, 4 \mathrm{H}), 1.77(\mathrm{dd}, J=6.8,1.5 \mathrm{~Hz}, 3 \mathrm{H}) ;{ }^{13} \mathrm{C} \mathrm{NMR}\left(125 \mathrm{MHz}, \mathrm{CDCl}_{3}\right) \delta 137.9$, 135.8, 130.4, 129.6, 128.4, 127.6, 127.0, 57.8, 18.1; MS (EI) $m / z$ (relative intensity) $264\left(\mathrm{M}^{+}, 50 \%\right), 173$ (100\%); Anal. Calcd for $\mathrm{C}_{18} \mathrm{H}_{20} \mathrm{~N}_{2}$ : C, 81.78; H, 7.62; N, 10.60. Found: C, 81.50; H, 7.58; N, 10.39.

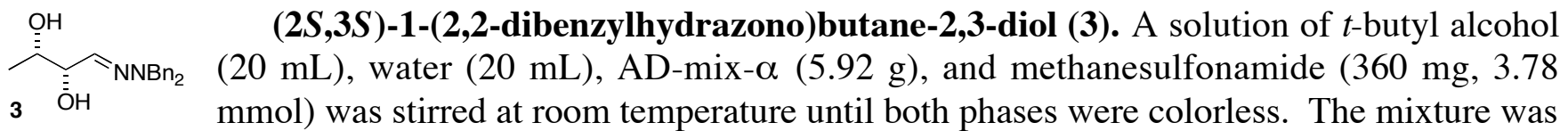
then cooled to $0^{\circ} \mathrm{C}$ (precipitation observed) and a solution of $2(1.0 \mathrm{~g}, 3.78 \mathrm{mmol})$ in $t$-butyl alcohol (5 $\mathrm{mL}$ ) was added. The reaction was allowed to warm to room temperature overnight. After $40 \mathrm{~h}$, the reaction was then quenched at $0{ }^{\circ} \mathrm{C}$ with sodium sulfite $(5.8 \mathrm{~g})$ and warmed to room temperature over 30 min. The solution was extracted with ethyl acetate $(3 \times 40 \mathrm{~mL})$, washed with $2 \mathrm{~N}$ aqueous $\mathrm{KOH}(2 \times 75$ $\mathrm{mL})$, dried $\left(\mathrm{Na}_{2} \mathrm{SO}_{4}\right)$, and concentrated. Flash chromatography $(5: 1$ petroleum ether/ethyl acetate $\rightarrow$ ethyl acetate) afforded syn-diol $\mathbf{3}(790 \mathrm{mg}, 70 \%)$ as a colorless oil. The enantiomeric purity was found to be $89 \%$ ee by HPLC (ChiralPak AD-H, 90:10 hexane/ $i$-PrOH; retention times: major 12.3 min, minor $18.4 \mathrm{~min}) . \quad[\alpha]_{\mathrm{D}}{ }^{22}+8.4\left(\mathrm{c} 0.27, \mathrm{CHCl}_{3}\right)$; IR (film, $\left.\mathrm{CHCl}_{3}\right) 3401,2972,1602,1495,1453,1357,1136$, 1073, $1028 \mathrm{~cm}^{-1} ;{ }^{1} \mathrm{H}$ NMR $\left(500 \mathrm{MHz}, \mathrm{CDCl}_{3}\right) \delta$ 7.35-7.25 (m, 10H), $6.52(\mathrm{~d}, J=3.1 \mathrm{~Hz}, 1 \mathrm{H}), 4.40(\mathrm{~s}$, $4 \mathrm{H}), 3.95(\mathrm{dd}, J=5.4,3.2 \mathrm{~Hz}, 1 \mathrm{H}), 3.62(\mathrm{~m}$, apparent quintet, $J=6.3 \mathrm{~Hz}, 1 \mathrm{H}) 3.52(\mathrm{br} \mathrm{s}, 1 \mathrm{H}), 2.60$ (br s, $1 \mathrm{H}), 1.08(\mathrm{~d}, J=6.4 \mathrm{~Hz}, 3 \mathrm{H}) ;{ }^{13} \mathrm{C}$ NMR $\left(125 \mathrm{MHz}, \mathrm{CDCl}_{3}\right) \delta 137.2,133.0,128.6,127.6,127.3,75.0$, 70.2, 58.2, 18.3; MS (CI) $\mathrm{m} / z$ (relative intensity) $299.5\left([\mathrm{M}+\mathrm{H}]^{+}, 70 \%\right), 281.3\left(\left[\mathrm{M}-\mathrm{H}_{2} \mathrm{O}\right]^{+}, 100 \%\right)$; Anal. Calcd for $\mathrm{C}_{18} \mathrm{H}_{22} \mathrm{~N}_{2} \mathrm{O}_{2}$ : C, 72.46; H, 7.43; N, 9.39. Found: C, 72.32; H, 7.43; N, 9.38.

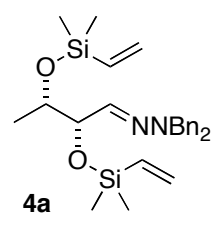

2-((2S,3S)-2,3-bis(dimethyl(vinyl)silyloxy)butylidene)-1,1-dibenzylhydrazine (4a). To a solution of $3(280 \mathrm{mg}, 0.938 \mathrm{mmol})$ in benzene $(10 \mathrm{~mL})$ was added triethylamine $(0.4 \mathrm{~mL}, 2.82 \mathrm{mmol})$, dimethylaminopyridine $(11 \mathrm{mg}, 0.094)$, and dimethylvinylchlorosilane $(0.4 \mathrm{~mL}, 2.82 \mathrm{mmol})$. After $30 \mathrm{~min}$, filtration through a plug of silica gel eluting with (3:1 hexane/ethyl acetate) afforded $4 \mathbf{a}(438 \mathrm{mg}, 100 \%)$ as a colorless oil. $[\alpha]_{\mathrm{D}}{ }^{22}+13.7\left(\mathrm{c} 0.98, \mathrm{CHCl}_{3}\right.$ ); IR (film) $3451,2958,1597,1453,1252,1069,836 \mathrm{~cm}^{-1} ;{ }^{1} \mathrm{H}$ NMR $\left(500 \mathrm{MHz}, \mathrm{CDCl}_{3}\right) \delta$ 7.30-7.27 (m, 4H), 7.26-7.21 (m, 6H), $6.33(\mathrm{~d}, J=6.7 \mathrm{~Hz}, 1 \mathrm{H}), 6.11(\mathrm{dd}, J=$ 20.3, $15.0 \mathrm{~Hz}, 1 \mathrm{H}), 6.10(\mathrm{dd}, J=20.3,14.8 \mathrm{~Hz}, 1 \mathrm{H}), 5.96(\mathrm{dd}, J=14.7,3.8 \mathrm{~Hz}, 1 \mathrm{H}), 5.95(\mathrm{dd}, J=14.8$, $3.9 \mathrm{~Hz}, 1 \mathrm{H}), 5.73(\mathrm{dd}, J=20.4,4.1 \mathrm{~Hz}, 1 \mathrm{H}), 5.71(\mathrm{dd}, J=20.3,4.0 \mathrm{~Hz}, 1 \mathrm{H}), 4.35(\mathrm{~s}, 4 \mathrm{H}), 4.02(\mathrm{dd}, J=$ $6.5,5.9 \mathrm{~Hz}, 1 \mathrm{H}) 3.71$ (dddd, apparent quintet, $J=6.3 \mathrm{~Hz}, 1 \mathrm{H}), 0.97(\mathrm{~d}, J=6.3 \mathrm{~Hz}, 3 \mathrm{H}), 0.14(\mathrm{~s}, 6 \mathrm{H})$, $0.13(\mathrm{~s}, 6 \mathrm{H}) ;{ }^{13} \mathrm{C}$ NMR $\left(125 \mathrm{MHz}, \mathrm{CDCl}_{3}\right) \delta 138.5,138.3,137.9,134.7,132.5,128.4,127.7,127.0,78.2$, 71.5, 57.6, 19.5, -1.3, -1.4; MS(CI) $\mathrm{m} / z$ (relative intensity) $468\left([\mathrm{M}+\mathrm{H}]^{+}, 25 \%\right), 366(45 \%), 338(100 \%)$ Anal. Calcd for $\mathrm{C}_{26} \mathrm{H}_{38} \mathrm{~N}_{2} \mathrm{O}_{2} \mathrm{Si}_{2}$ : C, 66.90; H, 8.21; N, 6.00. Found: C, 67.16; H, 8.32; N, 6.06.

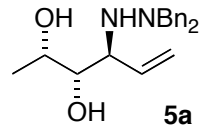

(2S,3S,4S)-4-(2,2-dibenzylhydrazinyl)hex-5-ene-2,3-diol (5a). To a solution of 4 a (328 $\mathrm{mg}, 0.703 \mathrm{mmol})$, and AIBN (58 $\mathrm{mg}, 0.351 \mathrm{mmol})$ in deoxygenated benzene (7 mL) was added thiophenol $(0.18 \mathrm{~mL}, 1.69 \mathrm{mmol})$. The mixture was heated at reflux for $20 \mathrm{~h}$. A solution of TBAF (1 M in THF, $2.8 \mathrm{~mL}, 2.8 \mathrm{mmol}$ ) was added and the mixture was allowed to cool to room temperature. After $24 \mathrm{~h}$, concentration and flash chromatography (5:1 petroleum ether/ethyl acetate) afforded $\mathbf{5 a}(181 \mathrm{mg}, 77 \%)$ as a pale yellow oil. [ $\alpha]_{\mathrm{D}}{ }^{23}-44.2$ (c 1.35, $\mathrm{CHCl}_{3}$ ); IR (film) 3406, 
3029, 2975, 2928, 1453, $992 \mathrm{~cm}^{-1} ;{ }^{1} \mathrm{H}$ NMR (500 MHz, $\mathrm{CDCl}_{3}$ ) $\delta$ 7.38-7.25 (m, 10H), 5.67 (ddd, apparent dt, $J=17.4,9.1 \mathrm{~Hz}, 1 \mathrm{H}), 5.18(\mathrm{dd}, J=10.4,1.2 \mathrm{~Hz}, 1 \mathrm{H}), 5.11(\mathrm{dd}, J=17.8,1.2 \mathrm{~Hz}, 1 \mathrm{H}), 3.82$ $(\mathrm{d}, J=12.9 \mathrm{~Hz}, 2 \mathrm{H}), 3.62(\mathrm{~d}, J=12.8 \mathrm{~Hz}, 2 \mathrm{H}), 3.63-3.57(\mathrm{~m}, 1 \mathrm{H}), 3.41-3.38(\mathrm{~m}, 2 \mathrm{H}), 3.00-2.60$ (br s, 2H), 2.80-2.40 (br s, 1H), $1.06(\mathrm{~d}, J=6.2 \mathrm{~Hz}, 3 \mathrm{H}) ;{ }^{13} \mathrm{C} \mathrm{NMR}\left(125 \mathrm{MHz}, \mathrm{CDCl}_{3}\right) \delta$ 137.2, 134.4, 129.8, 129.7, 128.4, 127.6, 118.7, 75.6, 67.7, 62.3, 60.7, 18.0; MS (CI) m/z (relative intensity) $327\left([\mathrm{M}+\mathrm{H}]^{+}\right.$, 21\%), 211 (100\%); Anal. Calcd for $\mathrm{C}_{20} \mathrm{H}_{26} \mathrm{~N}_{2} \mathrm{O}_{2}:$ C, 73.59; H, 8.03; N, 8.58. Found: C, 73.30; H, 7.95; N, 8.40 .

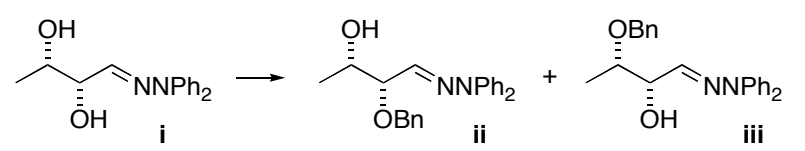

Differential O-Benzyl Protection of 2,3Dihydroxyhydrazone. A mixture of diol $\mathbf{i}^{1}(0.24 \mathrm{~g}$, $0.88 \mathrm{mmol})$ and $\mathrm{Bu}_{2} \mathrm{SnO}(0.23 \mathrm{~g}, 0.89 \mathrm{mmol})$ in benzene $(20 \mathrm{~mL})$ was heated at reflux for $4 \mathrm{~h}$. The solvent was replaced with toluene $(16 \mathrm{~mL})$, and $\mathrm{BnBr}(0.33 \mathrm{~mL}, 2.7 \mathrm{mmol})$ and TBAI $(98 \mathrm{mg}, 0.27$ mmol) were added. After heating at reflux for $24 \mathrm{~h}$, concentration and flash chromatography afforded the 2- $O$-benzyl isomer ii $(0.13 \mathrm{~g}, 0.37 \mathrm{mmol}, 42 \%)$ and 3- $O$-benzyl isomer iii $(51 \mathrm{mg}, 0.14 \mathrm{mmol}, 16 \%)$, both as pale yellow oils. Major isomer ii: $[\alpha]_{\mathrm{D}}{ }^{21}+9.3\left(\mathrm{c} 4.8, \mathrm{CHCl}_{3}\right)$; IR (film) $3455,1591,1496$, 1299, 1213, $1053 \mathrm{~cm}^{-1}$; ${ }^{1} \mathrm{H}$ NMR (500 MHz, $\mathrm{CDCl}_{3}$ ) $\delta$ 7.44-7.35 (m, 9H), 7.22-7.19 (m, 2H), 7.13-7.10 $(\mathrm{m}, 4 \mathrm{H}), 6.34(\mathrm{~d}, J=7.2 \mathrm{~Hz}, 1 \mathrm{H}), 4.67(\mathrm{~d}, J=11.9 \mathrm{~Hz}, 1 \mathrm{H}), 4.57(\mathrm{~d}, J=11.9 \mathrm{~Hz}, 1 \mathrm{H}), 3.93(\mathrm{dd}$, apparent triplet, $J=7.3 \mathrm{~Hz}, 1 \mathrm{H}), 3.80$ (dddd, apparent quintet, $J=6.5 \mathrm{~Hz}, 1 \mathrm{H}), 2.77(\mathrm{~s}, 1 \mathrm{H}), 1.18(\mathrm{~d}, J=$ $6.3 \mathrm{~Hz}, 3 \mathrm{H}) ;{ }^{13} \mathrm{C}$ NMR $\left(125 \mathrm{MHz}, \mathrm{CDCl}_{3}\right) \delta 143.6,138.2,135.3,130.0,128.5,128.0,127.8,124.7$, 122.3, 84.5, 71.0, 68.6, 18.5; MS (CI) $\mathrm{m} / z$ (relative intensity) $360\left(\mathrm{M}^{+}, 12 \%\right), 315$ (21\%), 169 (100\%); Anal. Calcd for $\mathrm{C}_{23} \mathrm{H}_{24} \mathrm{~N}_{2} \mathrm{O}_{2}$ : C, 76.64; H, 6.71; N, 7.77. Found: C, 76.82; H, 6.48; N, 7.61. Minor isomer iii. $[\alpha]_{\mathrm{D}}{ }^{19}-26.6$ (c $3.74, \mathrm{CHCl}_{3}$ ); IR (film) $3467,1591,1495,1298,1213,1091,1057 \mathrm{~cm}^{-1} ;{ }^{1} \mathrm{H}$ NMR (500 MHz, $\left.\mathrm{CDCl}_{3}\right) \delta$ 7.43-7.29 (m, 9H), 7.21-7.19 (m, 2H), 7.15-7.12 (m, 4H), $6.60(\mathrm{~d}, J=4.1$ $\mathrm{Hz}, 1 \mathrm{H}), 4.65(\mathrm{~d}, J=11.8 \mathrm{~Hz}, 1 \mathrm{H}), 4.50(\mathrm{~d}, J=11.8 \mathrm{~Hz}, 1 \mathrm{H}), 4.35$ (ddd, apparent quartet, $J=4.2 \mathrm{~Hz}$, $1 \mathrm{H}), 3.69$ (dddd, apparent quintet, $J=6.2 \mathrm{~Hz}, 1 \mathrm{H}), 3.35(\mathrm{~d}, J=4.2 \mathrm{~Hz}, 1 \mathrm{H}), 1.23(\mathrm{~d}, J=6.3 \mathrm{~Hz}, 3 \mathrm{H}) ;{ }^{13} \mathrm{C}$ NMR $\left(125 \mathrm{MHz}, \mathrm{CDCl}_{3}\right) \delta 143.6,138.3,136.8,129.7,128.3,127.6,124.4,122.3,76.7,74.2,71.0,15.3$; MS (CI) $m / z$ (relative intensity) $361\left([\mathrm{M}+\mathrm{H}]^{+}, 10 \%\right), 168(100 \%)$; Anal. Calcd for $\mathrm{C}_{23} \mathrm{H}_{24} \mathrm{~N}_{2} \mathrm{O}_{2}$ : C, 76.64; H, 6.71; N, 7.77. Found: C, 76.55; H, 6.72; N, 7.79.

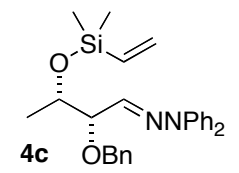

2-((2S,3S)-2-(benzyloxy)-3-(dimethyl(vinyl)silyloxy)butyl-idene)-1,1-

diphenylhydrazine (4c) To a solution of ii $(74.8 \mathrm{mg}, 0.208 \mathrm{mmol})$ in benzene $(5 \mathrm{~mL})$ was added triethylamine $(0.06 \mathrm{~mL}, 0.25 \mathrm{mmol})$ followed by dimethylaminopyridine (1 $\mathrm{mg})$. Dimethylvinylchlorosilane $(0.06 \mathrm{~mL}, 0.25 \mathrm{mmol})$ was added and the mixture was stirred overnight. Filtration through silica gel afforded $\mathbf{4 c}(84.7 \mathrm{mg}, 92 \%)$ as a colorless oil. $[\alpha]_{\mathrm{D}}^{22}=$ +16.9 (c 2.15, $\mathrm{CHCl}_{3}$ ); IR (film) 3042, 2970, 2885, 1592, 1495, 1084, $1055 \mathrm{~cm}^{-1}$; ${ }^{1} \mathrm{H} \mathrm{NMR}(500 \mathrm{MHz}$, $\left.\mathrm{CDCl}_{3}\right) \delta 7.40-7.05(\mathrm{~m}, 15 \mathrm{H}), 6.40(\mathrm{~d}, J=6.3 \mathrm{~Hz}, 1 \mathrm{H}), 6.08(\mathrm{ddd}, J=20.3,14.9,1.0 \mathrm{~Hz}, 1 \mathrm{H}), 5.95(\mathrm{dd}$, $J=14.9,4.0 \mathrm{~Hz}, 1 \mathrm{H}), 5.71(\mathrm{dd}, J=20.3,4.0 \mathrm{~Hz}, 1 \mathrm{H}), 4.58(\mathrm{ABq}, J=12.3 \mathrm{~Hz}, \Delta v=59.5 \mathrm{~Hz}, 2 \mathrm{H}), 3.95-$ $3.87(\mathrm{~m}, 2 \mathrm{H}), 1.11(\mathrm{~d}, J=5.4 \mathrm{~Hz}, 3 \mathrm{H}), 0.13(\mathrm{~s}, 3 \mathrm{H}), 0.12(\mathrm{~s}, 3 \mathrm{H}) ;{ }^{13} \mathrm{C} \mathrm{NMR}\left(125 \mathrm{MHz}, \mathrm{CDCl}_{3}\right) \delta 143.8$, 138.7, 138.1, 136.5, 132.8, 129.8, 128.2, 128.0, 127.4, 124.4, 122.4, 83.3, 70.8, 70.3, 20.1, -1.3, -1.5; MS (EI) $\mathrm{m} / z$ (relative intensity) $443\left(\mathrm{M}^{+}, 80 \%\right), 384(50 \%), 338$ (98\%), 316 (100\%); Anal. Calcd for $\mathrm{C}_{27} \mathrm{H}_{32} \mathrm{~N}_{2} \mathrm{O}_{2} \mathrm{Si}$ : C, 72.93; H, 7.25; N, 6.30. Found: C, 73.09; H, 7.23; N, 6.30.

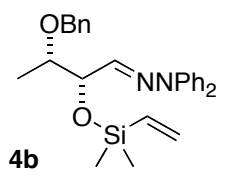

\section{2-((2S,3S)-3-(benzyloxy)-2-(dimethyl(vinyl)silyloxy)butyl-idene)-1,1-} diphenylhydrazine (4b). To a solution of iii $(47.5 \mathrm{mg}, 0.132 \mathrm{mmol})$ in benzene $(5 \mathrm{~mL})$ was added triethylamine $(0.04 \mathrm{~mL}, 0.16 \mathrm{mmol})$ followed by dimethylaminopyridine $(1$ $\mathrm{mg})$. Dimethylvinylchlorosilane $(0.04 \mathrm{~mL}, 0.16 \mathrm{mmol})$ was added and the mixture was 
stirred overnight. Filtration through silica gel afforded $\mathbf{4 b}\left(58.4 \mathrm{mg}\right.$, quant.) as a colorless oil. $[\alpha]_{\mathrm{D}}^{22}=$ -11.0 (c 3.56, $\mathrm{CHCl}_{3}$ ); IR (film) 3030, 2969, 2871, 1593, 1496, 1084, $1057 \mathrm{~cm}^{-1} ;{ }^{1} \mathrm{H} \mathrm{NMR}(500 \mathrm{MHz}$, $\left.\mathrm{CDCl}_{3}\right) \delta 7.44-7.10(\mathrm{~m}, 15 \mathrm{H}), 6.50(\mathrm{~d}, J=6.8 \mathrm{~Hz}, 1 \mathrm{H}), 6.18(\mathrm{dd}, J=20.3,14.9 \mathrm{~Hz}, 1 \mathrm{H}), 6.04(\mathrm{dd}, J=$ $14.9,3.9 \mathrm{~Hz}, 1 \mathrm{H}), 5.81(\mathrm{dd}, J=20.3,3.9 \mathrm{~Hz}, 1 \mathrm{H}), 4.62(\mathrm{~s}, 2 \mathrm{H}), 4.45(\mathrm{dd}, J=6.8,5.4 \mathrm{~Hz}, 1 \mathrm{H}), 3.61$ (pent, $J=6.4 \mathrm{~Hz}, 1 \mathrm{H}), 1.15(\mathrm{~d}, J=6.4 \mathrm{~Hz}, 3 \mathrm{H}), 0.25(\mathrm{~s}, 3 \mathrm{H}), 0.24(\mathrm{~s}, 3 \mathrm{H}) ;{ }^{13} \mathrm{C} \mathrm{NMR}\left(125 \mathrm{MHz}, \mathrm{CDCl}_{3}\right)$ $\delta$ 143.8, 138.8, 138.1, 137.8, 133.1, 129.7, 128.2, 127.7, 127.3, 124.2, 122.3, 77.3, 76.7, 71.5, 16.0, -1.4; MS (EI) $\mathrm{m} / \mathrm{z}$ (relative intensity) $444\left[\mathrm{M}^{+}\right](25 \%), 309(100 \%)$; Anal. Calcd for $\mathrm{C}_{27} \mathrm{H}_{32} \mathrm{~N}_{2} \mathrm{O}_{2} \mathrm{Si}$ : C, 72.93 ; H, 7.25; N, 6.30; Si, 6.32. Found: C, 73.04; H, 7.25; N, 6.25.

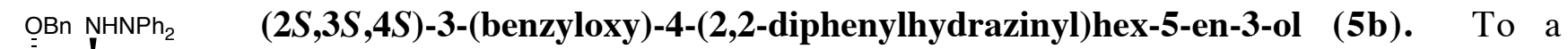
solution of hydrazone $4 \mathbf{b}(69 \mathrm{mg}, 0.155 \mathrm{mmol})$ in deoxygenated benzene $(2 \mathrm{~mL})$ was ÖH $\quad 5 \mathbf{b}$ added AIBN (5 mg, $0.03 \mathrm{mmol})$ and thiophenol $(0.02 \mathrm{~mL}, 0.19 \mathrm{mmol})$. The solution was heated at reflux for $16 \mathrm{~h}$. A solution of TBAF (1 M in THF, $1.6 \mathrm{~mL}, 1.6 \mathrm{mmol})$ was added and reflux was continued for $4 \mathrm{~h}$. Filtration through silica gel, concentration, and flash chromatography (10:1 to 3:1 Hex:EtOAc) afforded 5b (29 mg, 48\%, dr 94:6) as colorless oil. IR (film) 3540 (br), 3064, 2974, 2875, 1588, 1493, 1272, 1072, $913 \mathrm{~cm}^{-1} ;{ }^{1} \mathrm{H}$ NMR (300 MHz, $\left.\mathrm{CDCl}_{3}\right) \delta(\mathrm{ppm}) 7.39-6.93(\mathrm{~m}, 15 \mathrm{H}), 5.96$ $(\mathrm{ddd}, J=17.3,10.4,8.7 \mathrm{~Hz}, 1 \mathrm{H}), 5.23(\mathrm{dd}, J=10.4,1.7 \mathrm{~Hz}, 1 \mathrm{H}), 5.16(\mathrm{dd}, J=17.3,1.7 \mathrm{~Hz}, 1 \mathrm{H}), 4.55$ $(\mathrm{ABq}, J=11.5 \mathrm{~Hz}, \Delta v=44.9 \mathrm{~Hz}, 2 \mathrm{H}), 4.28($ br s, $1 \mathrm{H}), 3.77(\mathrm{~m}, 1 \mathrm{H}), 3.59-3.49(\mathrm{~m}, 2 \mathrm{H}), 2.77(\mathrm{~d}, J=2.7$ $\mathrm{Hz}, 1 \mathrm{H}), 1.09(\mathrm{~d}, J=6.2 \mathrm{~Hz}, 3 \mathrm{H}) ;{ }^{13} \mathrm{C} \mathrm{NMR}\left(75 \mathrm{MHz}, \mathrm{CDCl}_{3}\right) \delta(\mathrm{ppm}) 147.8,138.3,134.7,129.1$, $128.4,127.8,127.7,122.3,120.4,119.8,76.3,74.2,71.2,62.8,15.6 ; \mathrm{MS}$ (EI) $\mathrm{m} / z$ (relative intensity) $388\left[\mathrm{M}^{+}\right]$(7\%), 222 (20\%), $183(60 \%), 168(100 \%), 135(10 \%), 91(33 \%)$; Anal. Calcd for $\mathrm{C}_{25} \mathrm{H}_{28} \mathrm{~N}_{2} \mathrm{O}_{2}$ : C, 77.29; H, 7.26; N, 7.21. Found: C, 77.35; H, 7.21; N, 7.10.

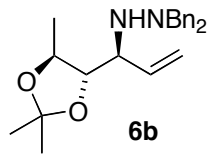

1,1-dibenzyl-2-((S)-1-((4S,5S)-2,2,5-trimethyl-1,3-dioxolan-4-yl)allyl)hydrazine (6b). To a solution of $\mathbf{5 a}(572 \mathrm{mg}, 1.75 \mathrm{mmol})$ in 2,2-dimethoxypropane (25 $\mathrm{mL})$ was added $p$-toluenesulfonic acid $(332 \mathrm{mg}, 1.75 \mathrm{mmol})$ and the mixture was stirred at room temperature for $12 \mathrm{~h}$. After completion, the reaction was concentrated and flash chromatography (10:1 to 5:1 petroleum ether/EtOAc) afforded $\mathbf{6 b}(628 \mathrm{mg}, 98 \%)$ as a colorless oil. $[\alpha]_{\mathrm{D}}{ }^{24}+20.4\left(\mathrm{c} 0.275, \mathrm{CHCl}_{3}\right)$; IR (film) 2360, 2341, 1454, 1377, 1243, $1088 \mathrm{~cm}^{-1}$; ${ }^{1} \mathrm{H} \mathrm{NMR}(500 \mathrm{MHz}$, $\left.\mathrm{CDCl}_{3}\right) \delta 7.36-7.26(\mathrm{~m}, 10 \mathrm{H}), 5.61(\mathrm{ddd}, J=17.4,10.3,8.5 \mathrm{~Hz}, 1 \mathrm{H}), 5.14(\mathrm{dd}, J=10.2,1.8 \mathrm{~Hz}, 1 \mathrm{H})$, $5.04(\mathrm{dd}, J=17.3,1.8 \mathrm{~Hz}, 1 \mathrm{H}), 3.81$ (dddd, apparent dq, $J=8.6,6.0 \mathrm{~Hz}, 1 \mathrm{H}), 3.78(\mathrm{~d}, J=13.1 \mathrm{~Hz}, 2 \mathrm{H})$, $3.65(\mathrm{~d}, J=12.9 \mathrm{~Hz}, 2 \mathrm{H}), 3.65(\mathrm{dd}, J=8.4,3.0 \mathrm{~Hz}, 1 \mathrm{H}), 3.22(\mathrm{dd}, J=8.6,3.0 \mathrm{~Hz}, 1 \mathrm{H}), 3.00-2.70$ (br s, $1 \mathrm{H}), 1.34(\mathrm{~s}, 3 \mathrm{H}), 1.31(\mathrm{~s}, 3 \mathrm{H}), 1.12(\mathrm{~d}, J=6.0 \mathrm{~Hz}, 3 \mathrm{H}) ;{ }^{13} \mathrm{C} \mathrm{NMR}\left(125 \mathrm{MHz}, \mathrm{CDCl}_{3}\right) \delta 138.2,136.4$, 129.6, 128.1, 127.1, 118.1, 107.8, 83.2, 72.9, 62.0, 61.2, 27.3, 26.8, 18.3; MS(CI) $\mathrm{m} / \mathrm{z}$ (relative intensity) $368\left([\mathrm{M}+\mathrm{H}]^{+}, 70 \%\right)$; Anal. Calcd for $\mathrm{C}_{23} \mathrm{H}_{30} \mathrm{~N}_{2} \mathrm{O}_{2}$ : C, 75.37; H, 8.25; N, 7.64. Found: C, 74.97; H, 8.21; N, 7.37 .

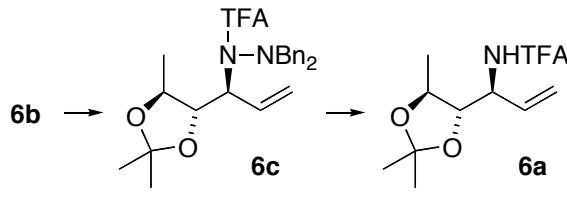

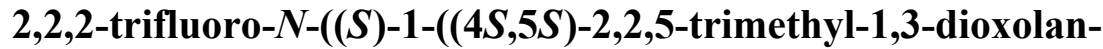
4-yl)allyl)acetamide (6a). To a solution of $\mathbf{6 b}(272 \mathrm{mg}, 0.743$ $\mathrm{mmol})$ in tetrahydrofuran $(10 \mathrm{~mL})$ at $-78{ }^{\circ} \mathrm{C}$ was added $n$-BuLi $(1.2$ $\mathrm{M}$ in hexanes, $0.65 \mathrm{~mL}, 0.78 \mathrm{mmol})$. The red solution was stirred 1 h. Freshly distilled trifluoroacetic anhydride $(0.31 \mathrm{~mL}, 2.2 \mathrm{mmol})$ was added slowly at $-78{ }^{\circ} \mathrm{C}$, and the reaction was warmed to room temperature. After $16 \mathrm{~h}$, the reaction mixture was diluted with diethyl ether $(20 \mathrm{~mL})$, washed with saturated aqueous sodium bicarbonate $(2 \mathrm{x}$ $20 \mathrm{~mL}$ ), dried and concentrated. Flash chromatography (20:1 hexane/ethyl acetate) afforded 6c (345 $\mathrm{mg}, 100 \%)$ as a yellow oil. To a solution of $\mathbf{6 c}(268 \mathrm{mg}, 0.580 \mathrm{mmol})$ in deoxygenated methanol $(1.2$ $\mathrm{mL}$ ) was added samarium diiodide (ca. $0.3 \mathrm{M}$ in THF, $15.5 \mathrm{~mL}$, ca. $4.6 \mathrm{mmol}$ ) dropwise until the color 
remained blue. The reaction mixture was concentrated followed by purification by flash chromatography (10:1 to 1:1 petroleum ether:EtOAc) to afford $\mathbf{6 a}(155 \mathrm{mg}, 100 \%)$ as a colorless oil. $[\alpha]_{\mathrm{D}}{ }^{27}-2.63$ (c 0.495, $\mathrm{CHCl}_{3}$ ); IR (film) 3300, 1708, 1554, 1379, 1214, $1173 \mathrm{~cm}^{-1} ;{ }^{1} \mathrm{H} \mathrm{NMR}(500 \mathrm{MHz}$, $\left.\mathrm{CDCl}_{3}\right) \delta 6.64(\mathrm{~d}, J=8.4 \mathrm{~Hz}, 1 \mathrm{H}), 5.87(\mathrm{ddd}, J=17.2,10.4,7.7 \mathrm{~Hz}, 1 \mathrm{H}), 5.37(\mathrm{~d}, J=10.4 \mathrm{~Hz}, 1 \mathrm{H}), 5.35$ $(\mathrm{d}, J=17.2 \mathrm{~Hz}, 1 \mathrm{H}), 4.55$ (ddd, apparent dt, $J=8.4,3.3 \mathrm{~Hz}, 1 \mathrm{H}), 3.90$ (dddd, apparent dq, $J=8.5,6.0$ $\mathrm{Hz}, 1 \mathrm{H}), 3.68(\mathrm{dd}, J=8.5,3.3 \mathrm{~Hz}, 1 \mathrm{H}), 1.42(\mathrm{~s}, 3 \mathrm{H}), 1.37(\mathrm{~s}, 3 \mathrm{H}), 1.30(\mathrm{~d}, J=6.0 \mathrm{~Hz}, 3 \mathrm{H}) ;{ }^{13} \mathrm{C} \mathrm{NMR}$ $\left(125 \mathrm{MHz}, \mathrm{CDCl}_{3}\right) \delta 157.0\left(\mathrm{q},{ }^{2} J_{\mathrm{CF}}=45 \mathrm{~Hz}\right), 131.2,120.4,116.0\left(\mathrm{q},{ }^{1} J_{\mathrm{CF}}=298 \mathrm{~Hz}\right), 109.0,82.7,73.4$, 53.0, 27.3, 26.6, 17.5; MS (CI) $\mathrm{m} / z$ (relative intensity) $268.3\left([\mathrm{M}+\mathrm{H}]^{+}, 100 \%\right), 114(22 \%)$.

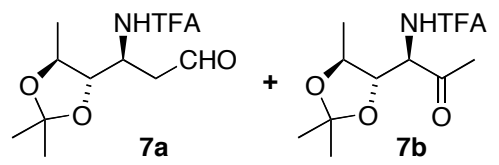

Wacker Oxidations (see Scheme 5). To a solution of $\mathbf{6 a}, \mathbf{6 b}$, or $\mathbf{6 c}$ (ca. 0.1-0.2 mmol) in dimethylacetamide (or DMF) and water (7:1, 0.05 M) was added the copper salt indicated in Scheme 5 (2 equiv) followed by $\mathrm{PdCl}_{2}$ (ca. $\left.10 \mathrm{~mol} \%\right)$. The mixture was stirred under $\mathrm{O}_{2}(1 \mathrm{~atm}$,

balloon) for 1.5-4 d. The reaction was filtered through Celite, diluted with diethyl ether $(10 \mathrm{~mL})$, washed with saturated aqueous sodium bicarbonate $(2 \times 10 \mathrm{~mL})$, dried, and concentrated. Purification by flash chromatography (10:1 to $1: 1$ petroleum ether/EtOAc) afforded $7 \mathbf{a}$ and $\mathbf{7 b}$ in the yields and ratios shown in Scheme 5. 2,2,2-Trifluoro- $N-((S)-3-0 x 0-1-((4 S, 5 S)-2,2,5-t r i m e t h y l-1,3-d i o x o l-a n-4-$ yl)propyl) acetamide (7a): Colorless solid; mp 42-43 ${ }^{\circ} \mathrm{C}$; $[\alpha]_{\mathrm{D}}{ }^{25}-7.44$ (c 1.56, $\mathrm{CHCl}_{3}$ ); IR (film) 3304, 2917, 1708, 1558, 1381, 1169, $858 \mathrm{~cm}^{-1} ;{ }^{1} \mathrm{H}$ NMR (500 MHz, $\left.\mathrm{CDCl}_{3}\right) \delta 9.79(\mathrm{~s}, 1 \mathrm{H}), 7.22(\mathrm{~d}, J=7.1$ $\mathrm{Hz}, 1 \mathrm{H}), 4.43-4.37$ (m, 1H), 3.91 (ddd, apparent dq, $J=7.6,6.0 \mathrm{~Hz}, 1 \mathrm{H}), 3.69$ (dd, apparent triplet, $J=$ $7.5 \mathrm{~Hz}, 1 \mathrm{H}), 3.05$ (dd, $J=18.3,4.9 \mathrm{~Hz}, 1 \mathrm{H}), 2.78(\mathrm{dd}, J=18.3,4.9 \mathrm{~Hz}, 1 \mathrm{H}), 1.38(\mathrm{~s}, 3 \mathrm{H}), 1.37(\mathrm{~s}, 3 \mathrm{H})$, $1.30(\mathrm{~d}, J=6.0 \mathrm{~Hz}, 3 \mathrm{H}) ;{ }^{13} \mathrm{C} \mathrm{NMR}\left(125 \mathrm{MHz}, \mathrm{CDCl}_{3}\right) \delta 200.2,156.8\left(\mathrm{q},{ }^{2} J_{\mathrm{CF}}=37 \mathrm{~Hz}\right), 115.6\left(\mathrm{q},{ }^{1} J_{\mathrm{CF}}=\right.$ $286 \mathrm{~Hz}), 109.0,81.8,75.4,47.8,43.1,27.2$, 26.8, 18.4; MS (CI) m/z (relative intensity) $284\left([\mathrm{M}+\mathrm{H}]^{+}\right.$, $70 \%$ ), 240 (21\%), 226 (100\%); Anal. Calcd for $\mathrm{C}_{11} \mathrm{H}_{16} \mathrm{~F}_{3} \mathrm{NO}_{4}$ : C, 46.64; H, 5.69; N, 4.95. Found: C,

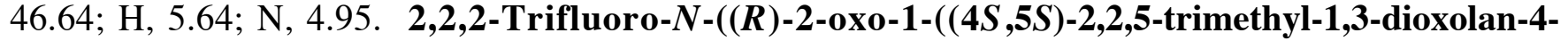
yl)propyl) acetamide (7b): Yellow oil; $[\alpha]_{\mathrm{D}}{ }^{24}=-32.5^{\circ}$ (c $0.195, \mathrm{CDCl}_{3}$ ); IR (film) 3321 (br), 2925 , 1721, 1710, 1213, $1170 \mathrm{~cm}^{-1} ;{ }^{1} \mathrm{H}$ NMR $\left(300 \mathrm{MHz} \mathrm{CDCl}_{3}\right) \delta$ 7.18-7.10 (s, 1H), 4.79 (dd, J = 7.57, 7.57 $\mathrm{Hz}, 1 \mathrm{H}), 4.19(\mathrm{dd}, J=8.01,5.93 \mathrm{~Hz}, 1 \mathrm{H}), 3.53(\mathrm{dd}, J=7.96,7.07 \mathrm{~Hz}, 1 \mathrm{H}), 2.45-2.35(\mathrm{~s}, 3 \mathrm{H}), 1.41(\mathrm{~d}, J$ $=5.72 \mathrm{~Hz}, 6 \mathrm{H}), 1.26(\mathrm{~d}, J=5.96 \mathrm{~Hz}, 3 \mathrm{H}) ;{ }^{13} \mathrm{C} \mathrm{NMR}\left(125 \mathrm{MHz}, \mathrm{CDCl}_{3}\right) \delta 203.7,109.4,81.9,76.7,59.4$, 29.9, 27.1, 26.5, 18.3 (TFA quartets were obscured in baseline noise); MS (EI) $\mathrm{m} / z$ (relative intensity) $283\left(\mathrm{M}^{+}, 1 \%\right), 269$ (10\%), 268 (100\%); HRMS: Calcd for $\mathrm{C}_{11} \mathrm{H}_{16} \mathrm{~F}_{3} \mathrm{NO}_{4}$ : 283.1031; Found: 283.1030.

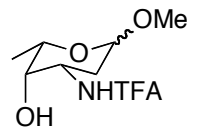

Methyl $N$-Trifluoroacetyl-L-daunosaminide. A solution of aldehyde 7a $(31.4 \mathrm{mg}$, $0.11 \mathrm{mmol})$ in anhydrous $\mathrm{HCl}$ solution $(0.1 \mathrm{M}$ in methanol, $4.4 \mathrm{~mL}, 0.44 \mathrm{mmol})$ was stirred for $6 \mathrm{~h}$ at room temperature. The reaction mixture was diluted with ether, washed by saturated $\mathrm{NaHCO}_{3}$ solution and dried through $\mathrm{Na}_{2} \mathrm{SO}_{4}$. Concentration and column chromatography afforded 8 as a mixture of $\alpha$ - and $\beta$-anomers $\left(28 \mathrm{mg}, 100 \%, \alpha / \beta=3: 1^{2}\right)$. Spectroscopic properties of the mixture $\mathbf{8 a} / \mathbf{8 b}$ matched the previously reported data. ${ }^{3}$

\section{References and Notes}

\footnotetext{
${ }^{1}$ Friestad, G.K.; Massari, S.E. J. Org. Chem. 2004, 69, 863-875.

${ }^{2}$ After prolonged exposure to $\mathrm{TsOH} / \mathrm{MeOH}$ over $5 \mathrm{~d}$, the $\alpha$-anomer was obtained.

${ }^{3}$ (a) Shelton, C. J.; Harding, M. M. J. Chem. Research (S) 1995, 158. (b) Shelton, C. J.; Harding, M. M. J. Chem. Research (M) 1995, 1201-1219. We thank Dr. M. Harding for kindly providing copies of the data.
} 
NMR spectra of 2
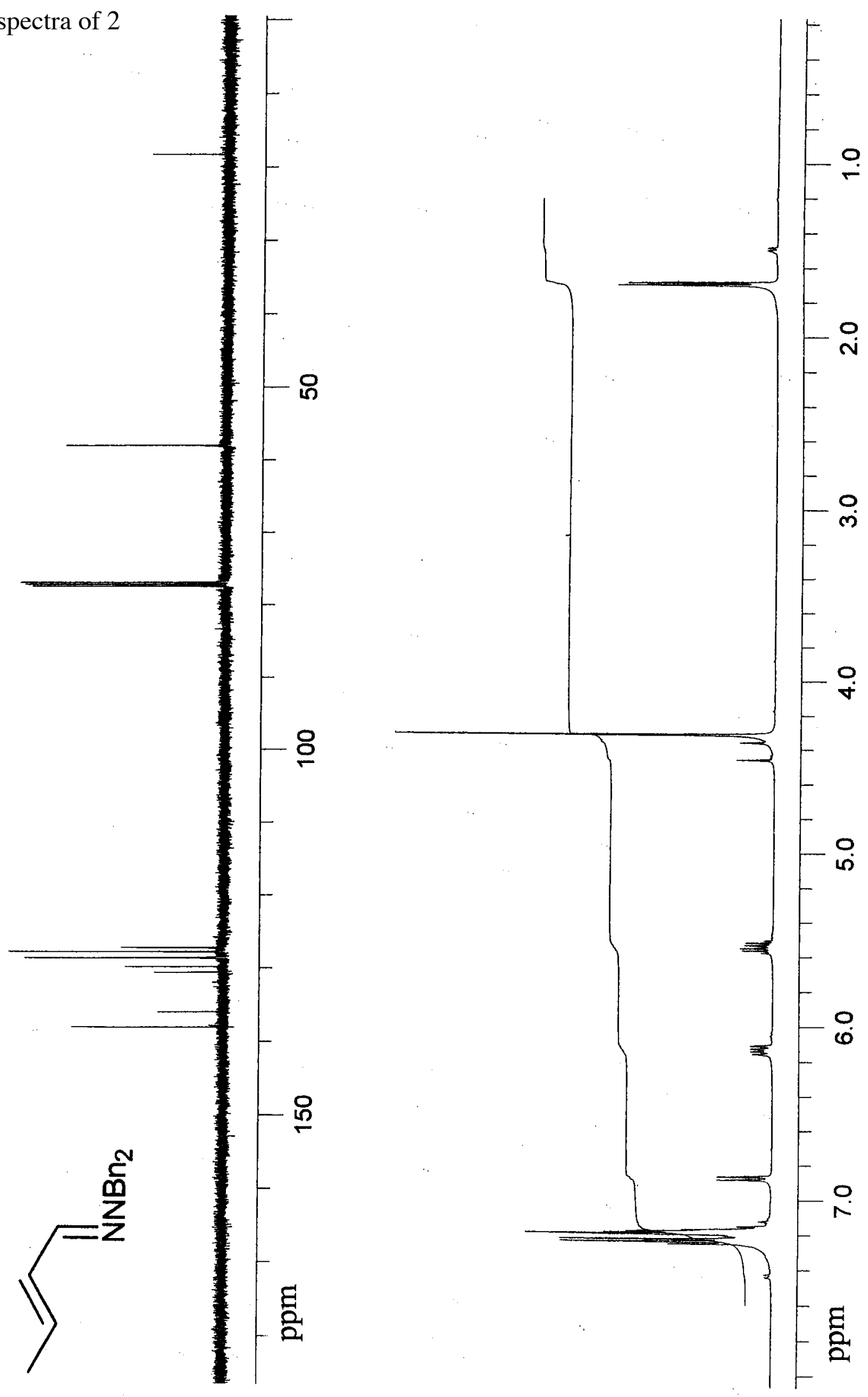
NMR spectra of 3
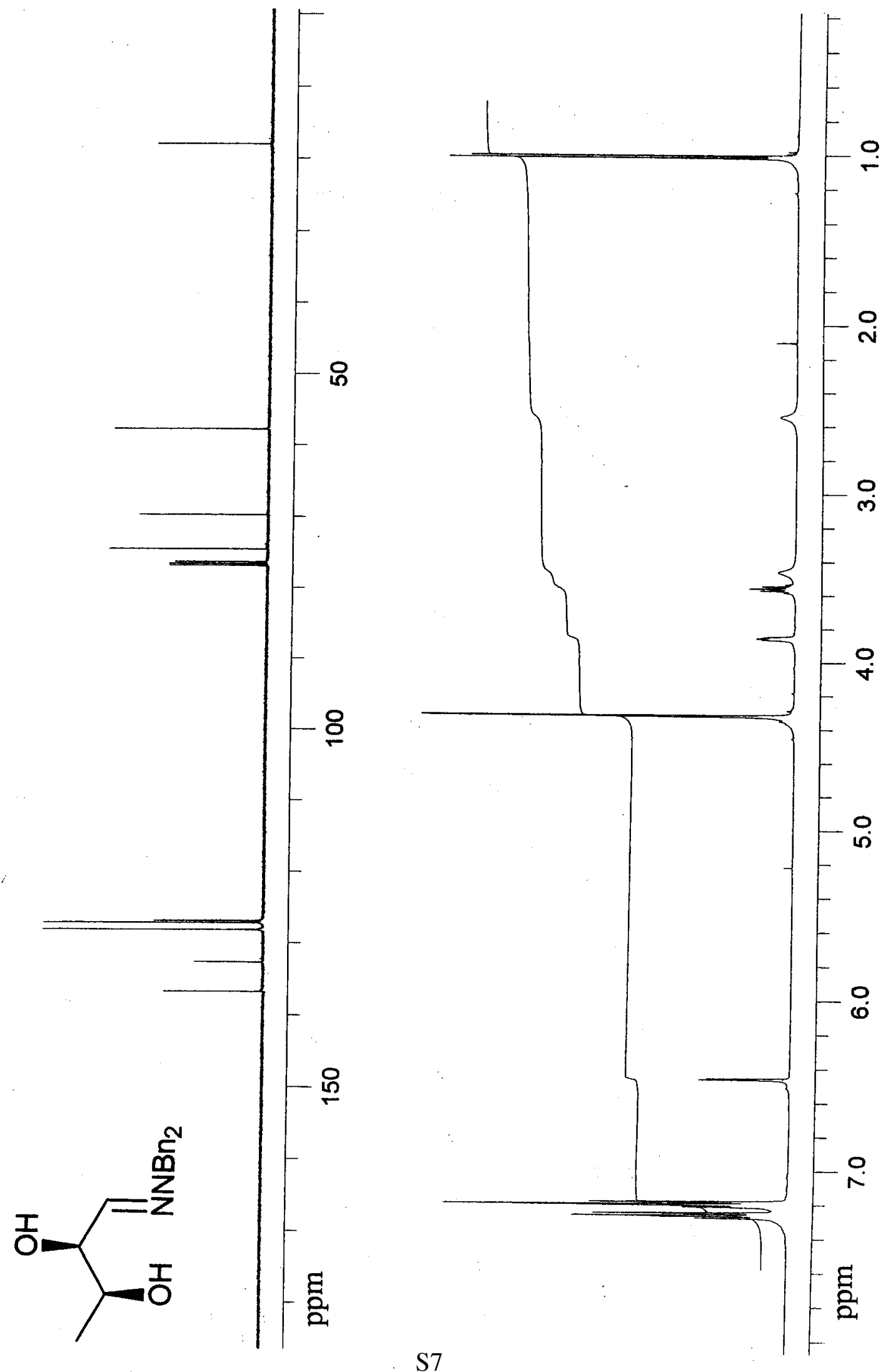


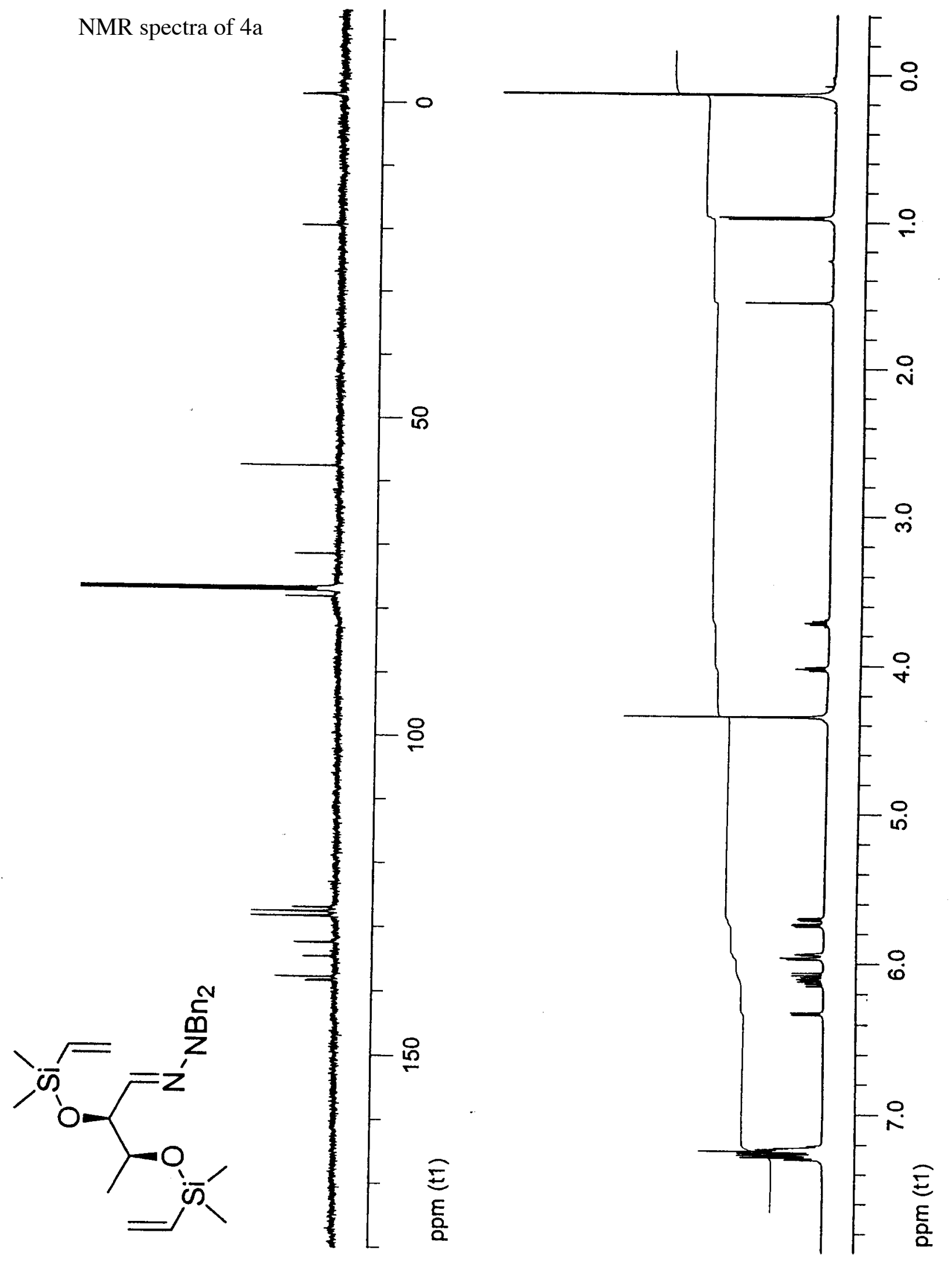




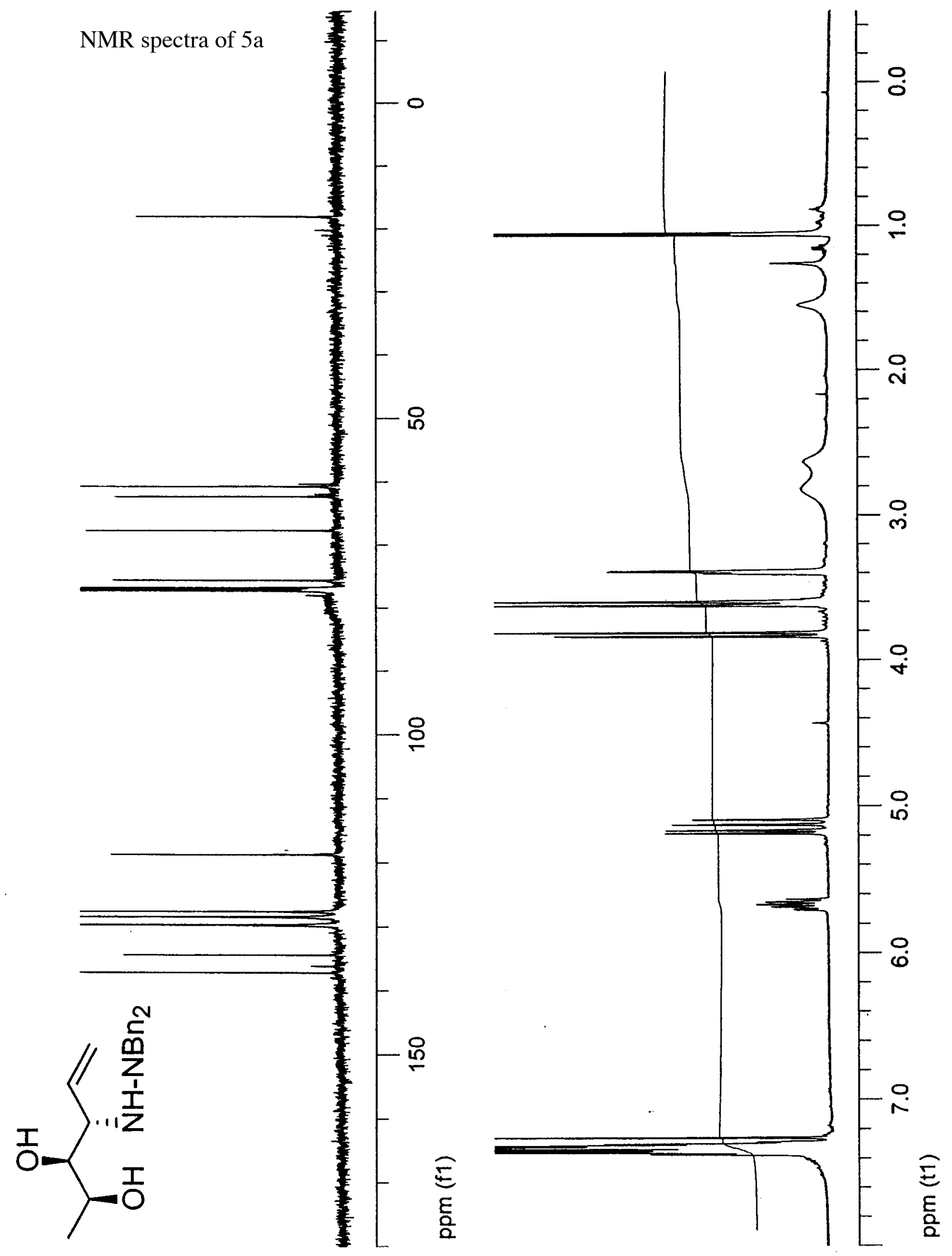




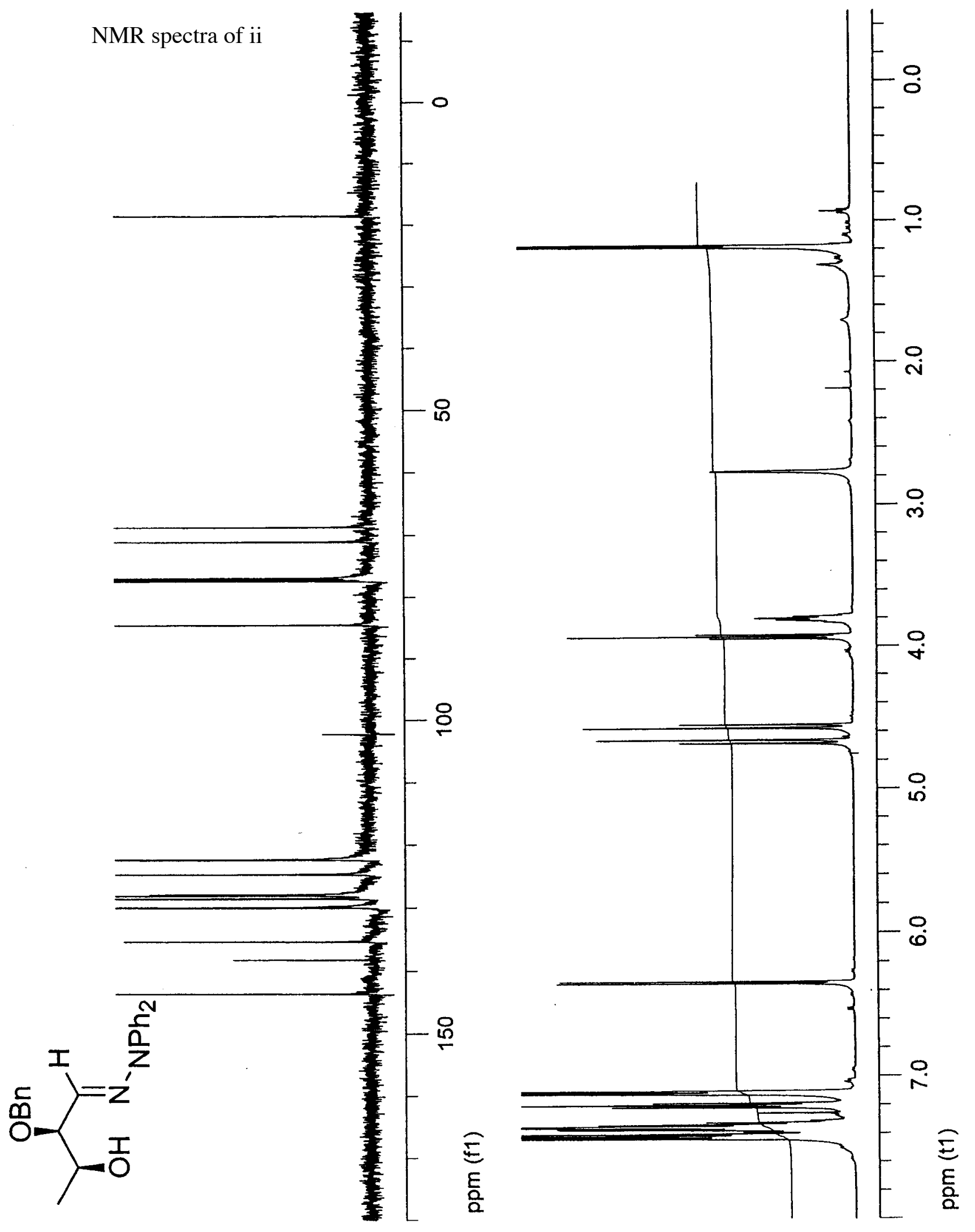




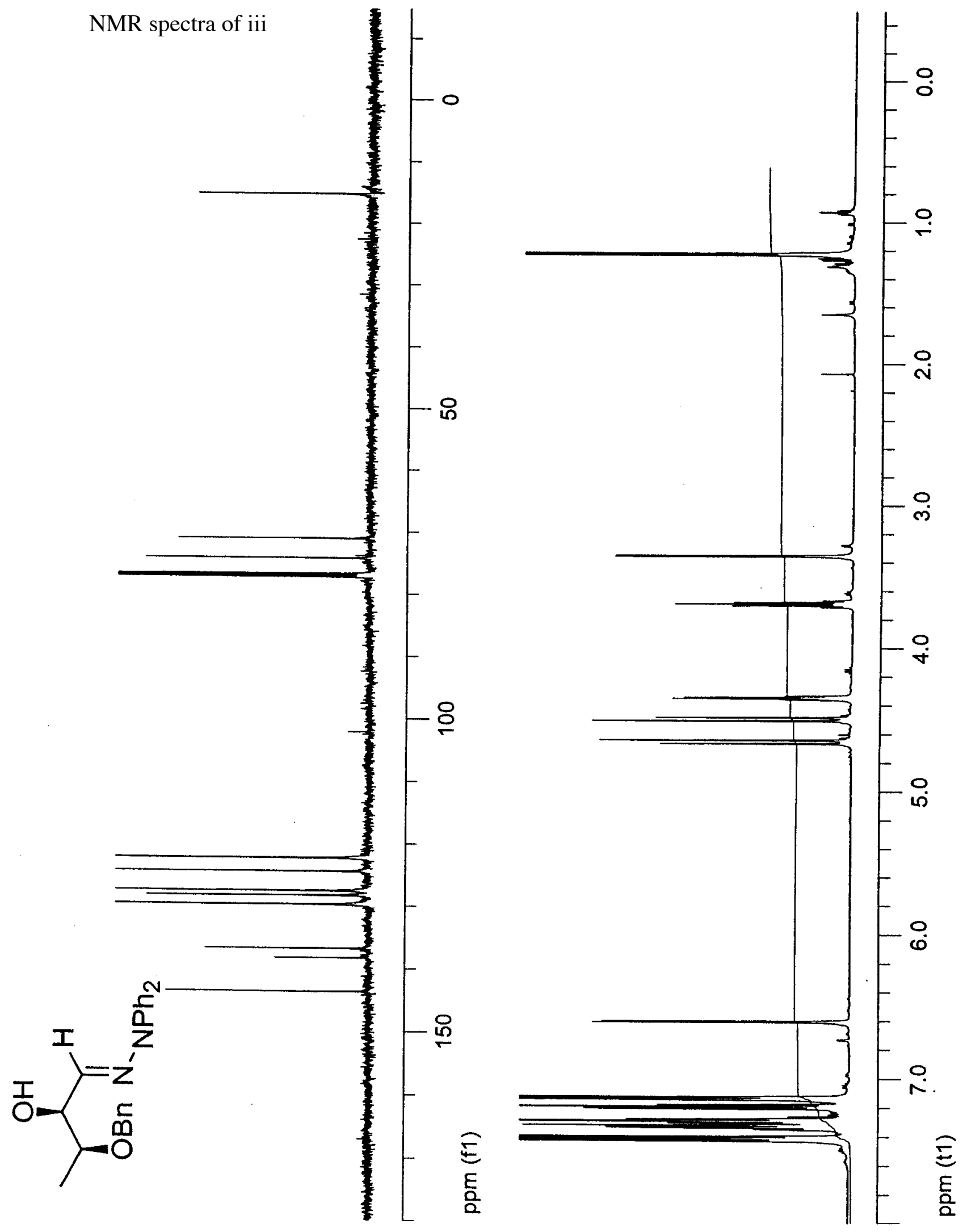




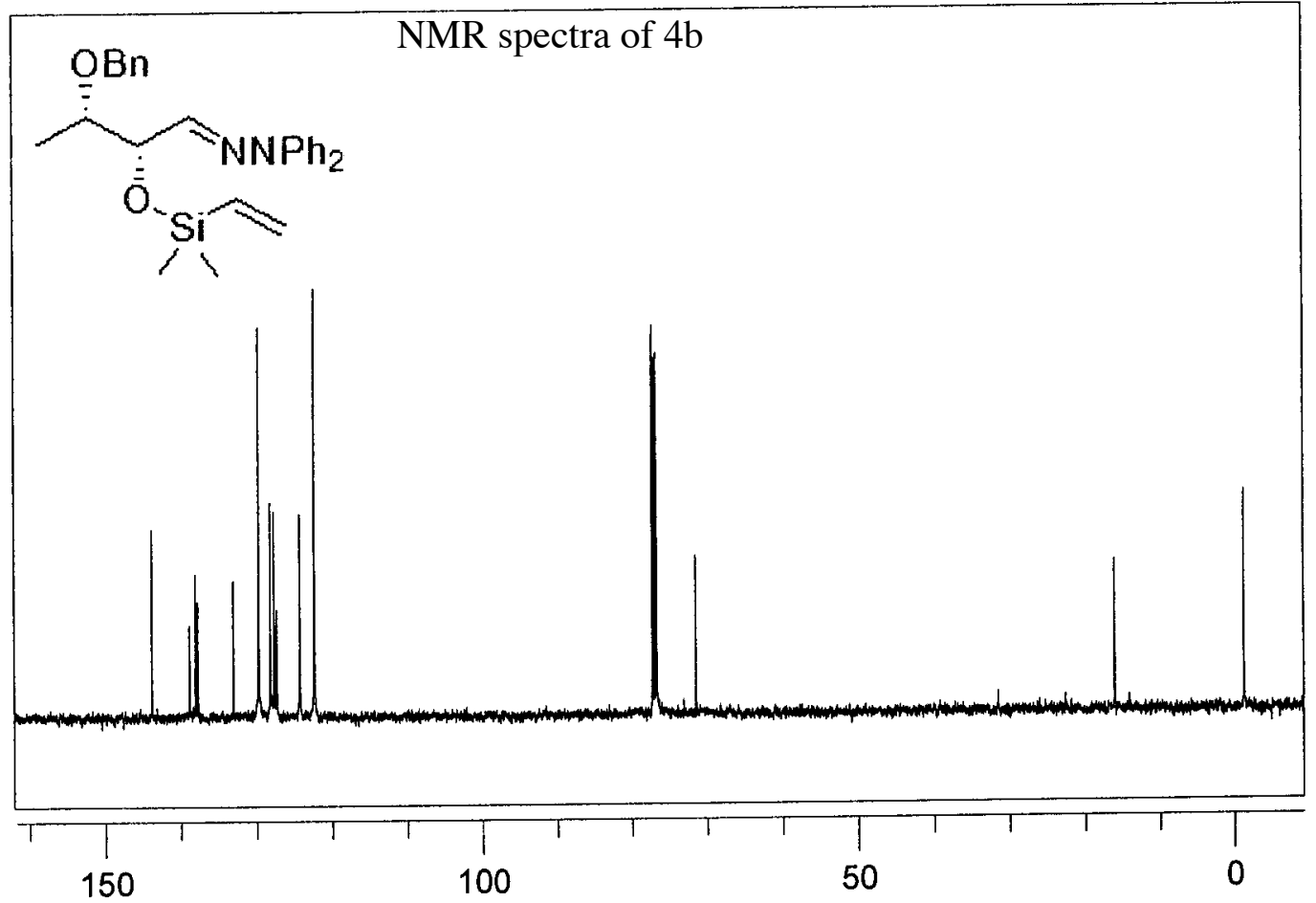

ppm (t1)

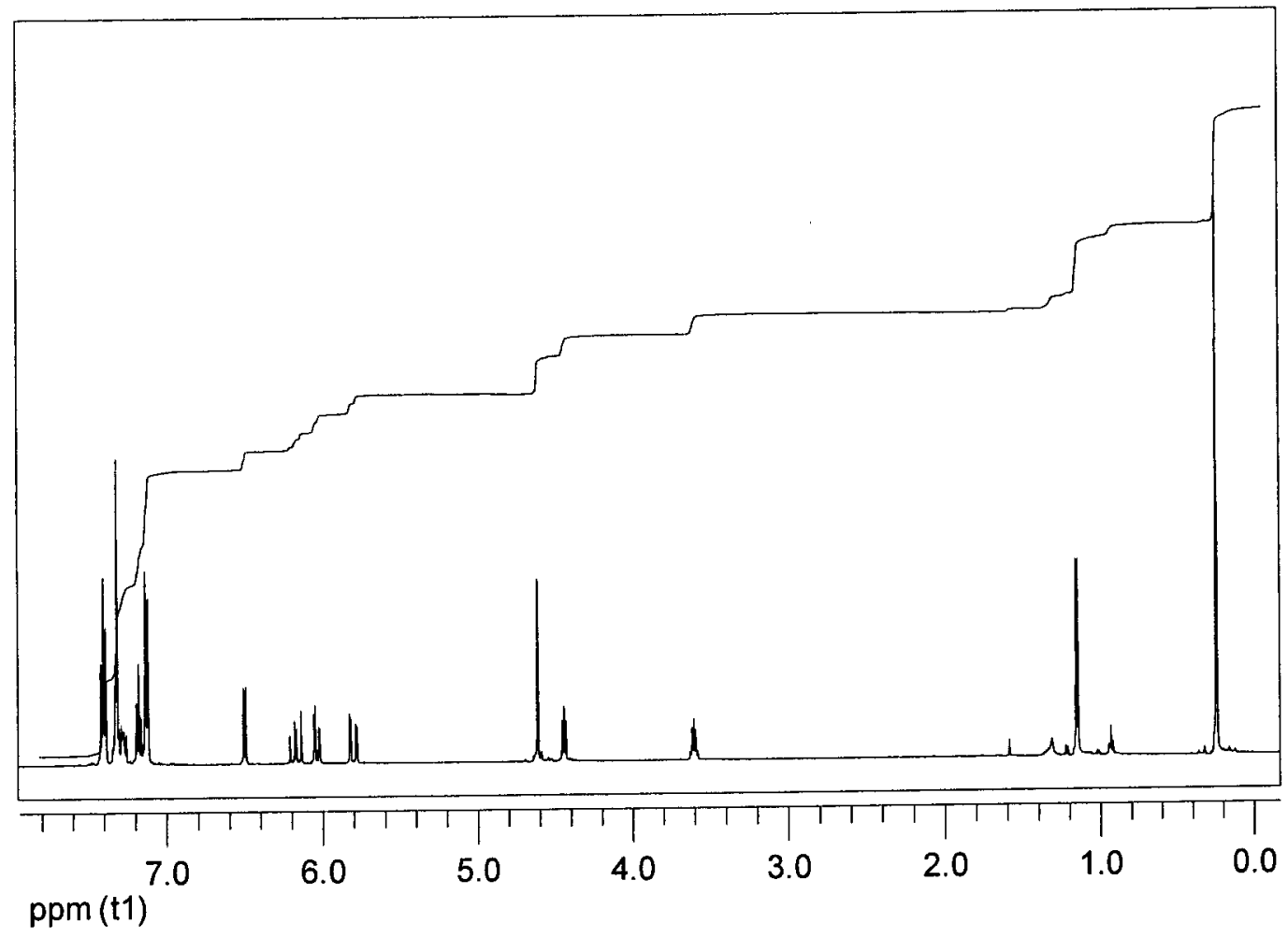



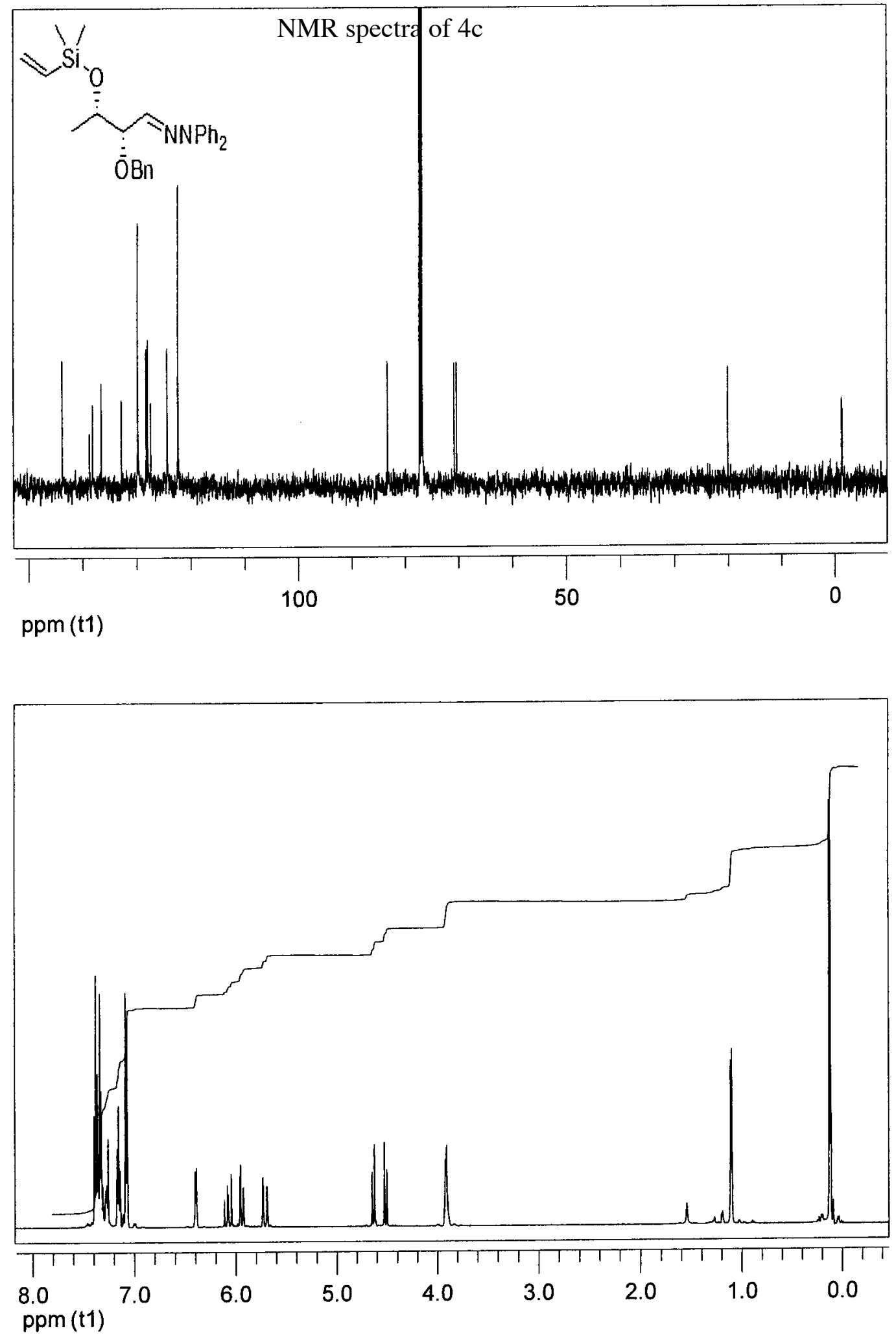

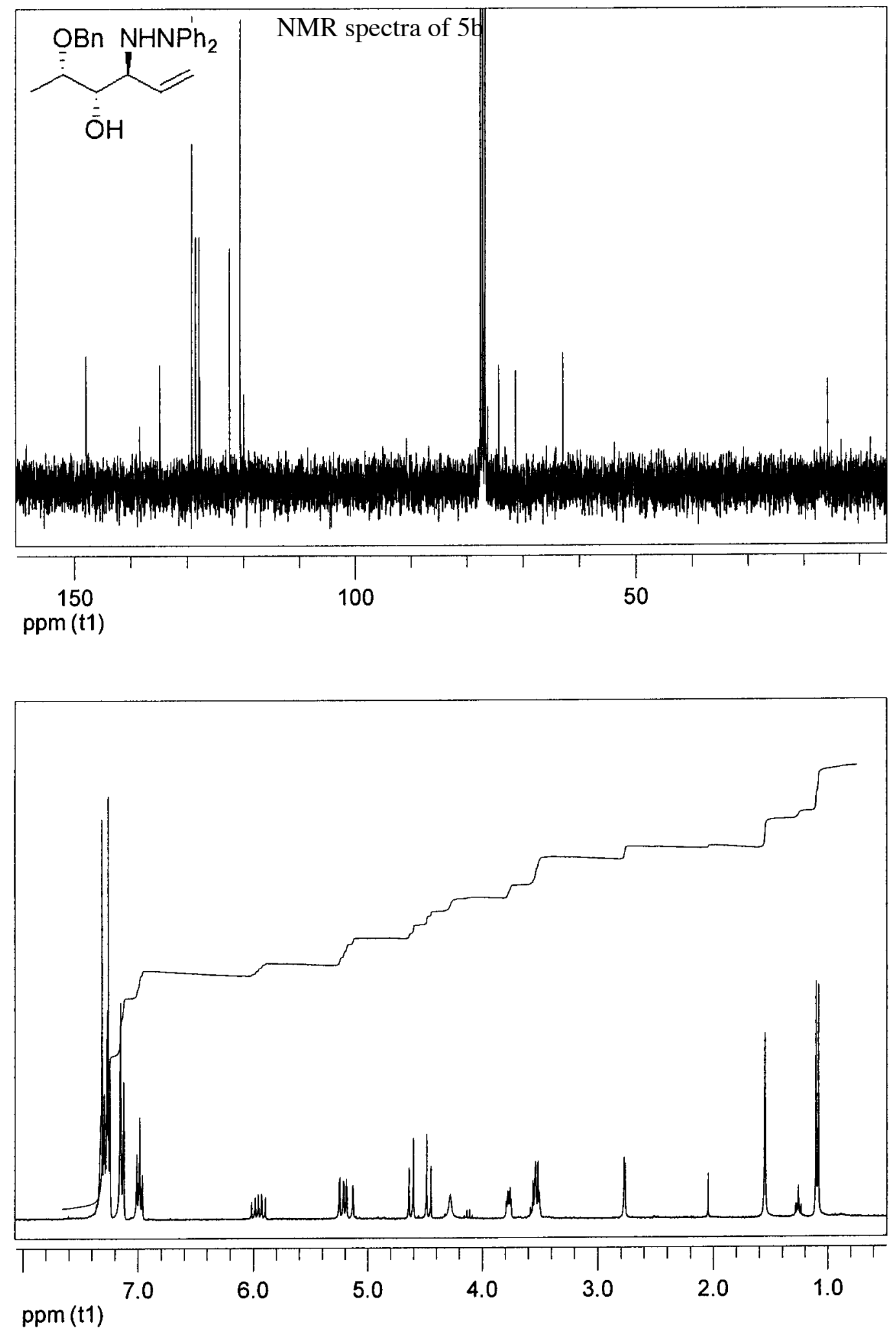


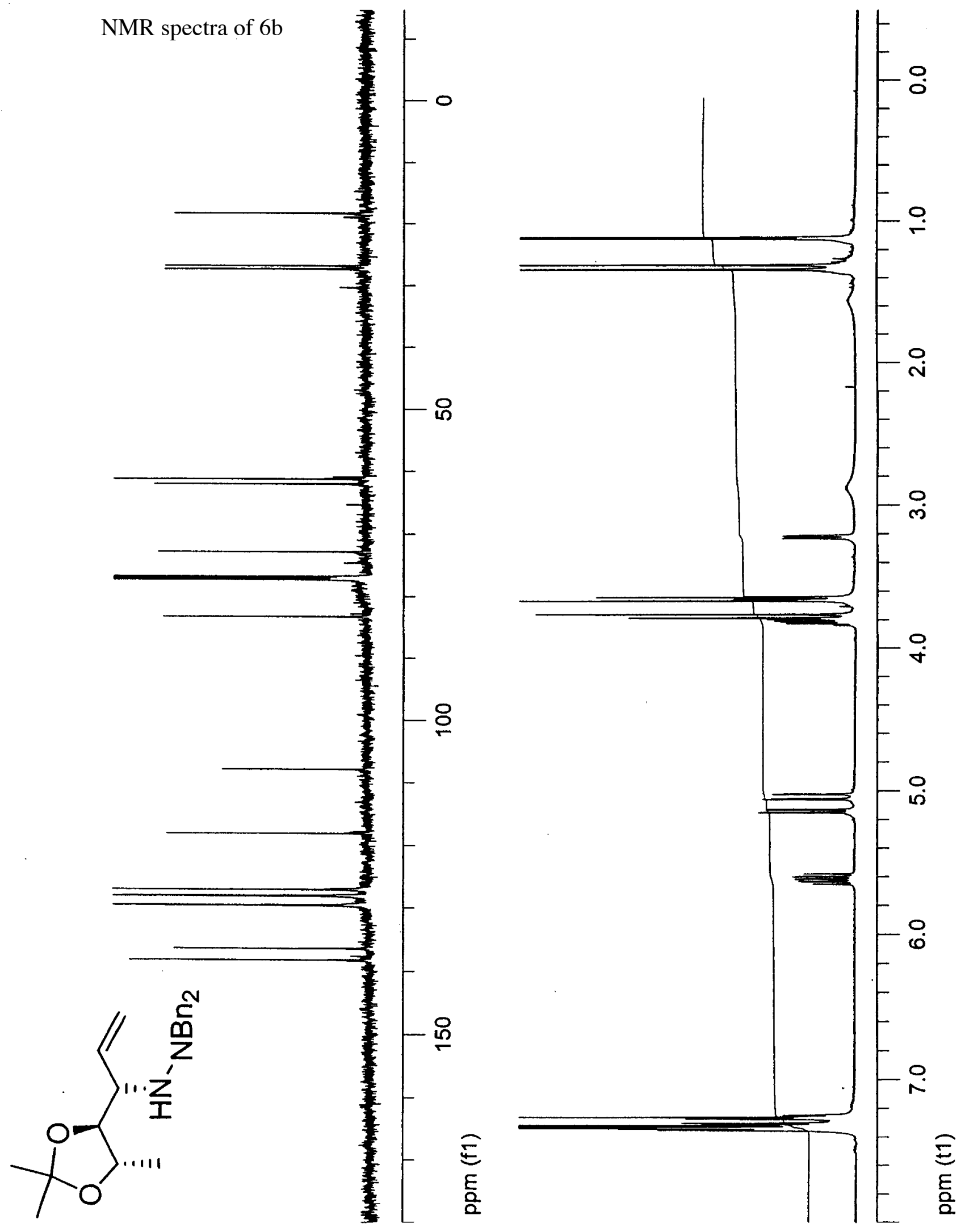




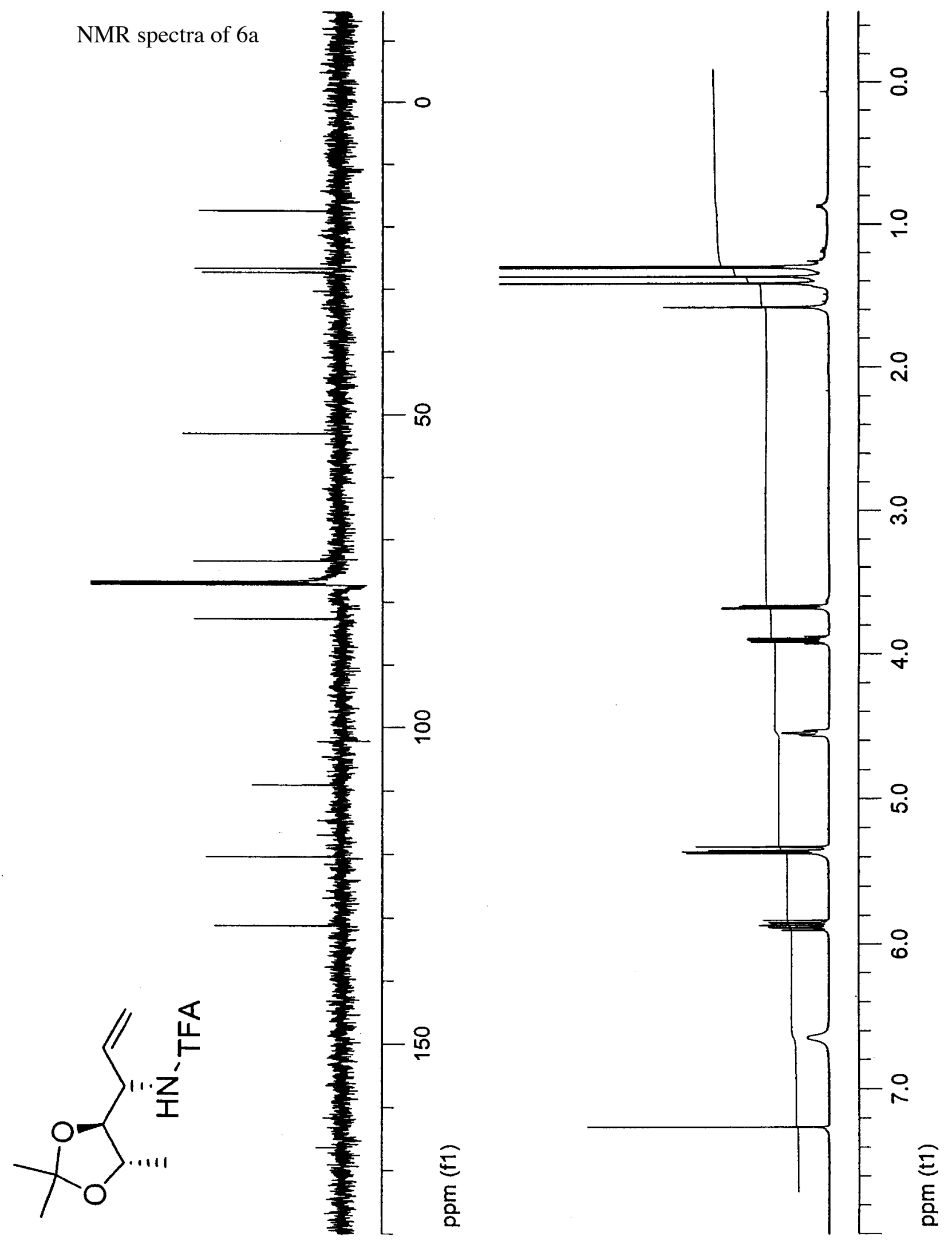



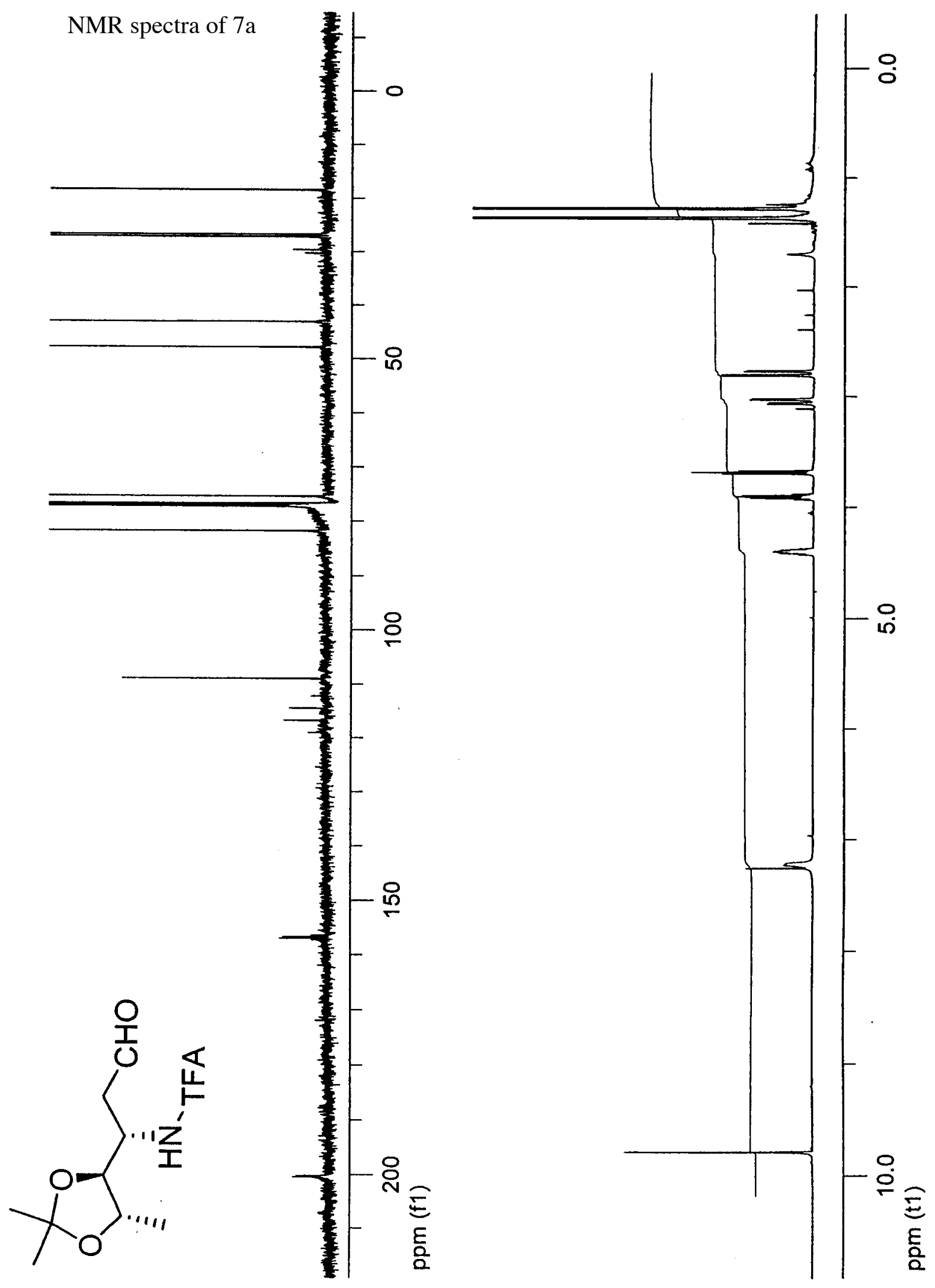


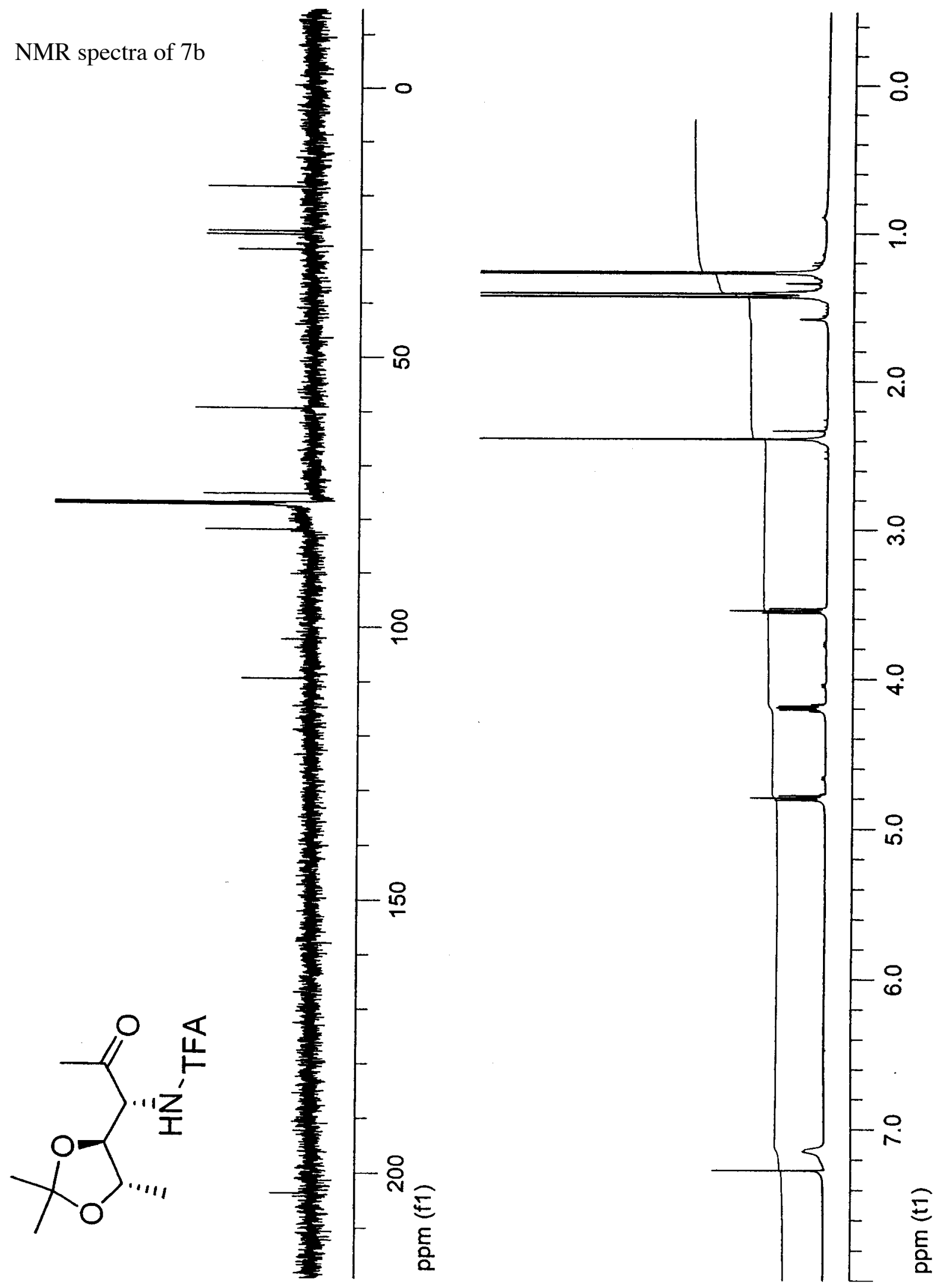


NMR spectrum of 8 (alpha anomer)

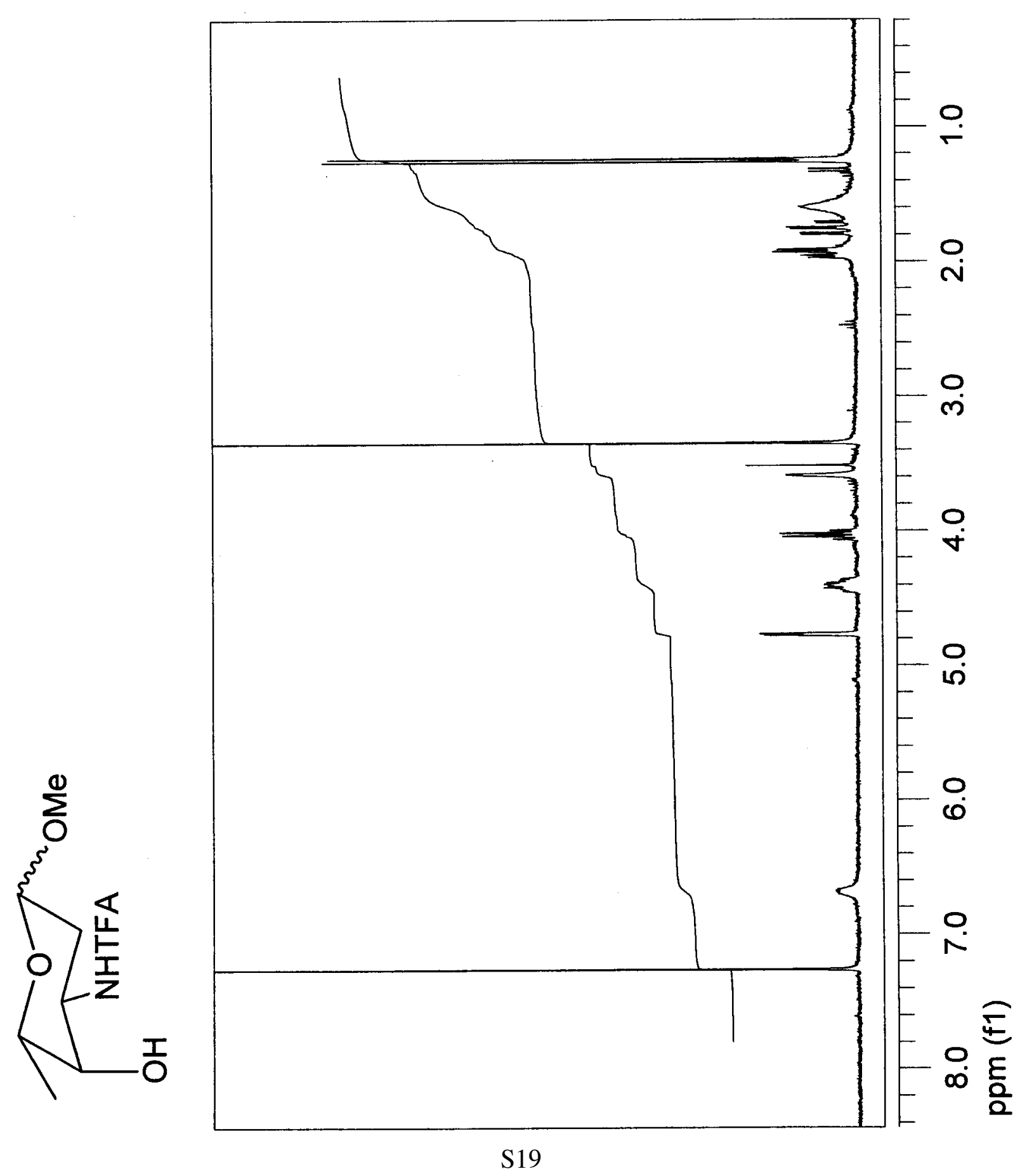




\section{Comparison of Selected Daunosamine Syntheses}

Some notable syntheses of daunosamine derivatives from achiral and/or non-carbohydrate precursors are shown in the table below. Syntheses longer than nine steps are not included. Racemic syntheses are not included except where an asymmetric adaptation is noted by the authors. To make the comparisons with the present work as valid as possible, the number of steps and overall yield are calculated from commercial materials to the daunosamine $N$-acyl glycoside derivatives whenever possible, even if subsequent hydrolytic conversions to daunosamine itself are described. When starting materials are non-commercial, the yields and steps are calculated by incorporating the data from the preparations cited by the authors.

\begin{tabular}{|c|c|c|c|c|}
\hline Lead author & reference & target & $\begin{array}{l}\text { yield } \\
\text { (steps) } \\
\end{array}$ & $\begin{array}{l}\text { starting material } \\
\text { (key steps) }\end{array}$ \\
\hline \multicolumn{5}{|c|}{ A. Racemic syntheses } \\
\hline Danishefsky & $\begin{array}{l}\text { J. Am. Chem. Soc. } \\
\text { 1985, 107, } 1269\end{array}$ & $\begin{array}{l}\text { methyl } N, O \text { - } \\
\text { diacetyldaunosaminide } \\
\text { (racemic) }\end{array}$ & $\begin{array}{l}15.4 \% \\
\text { (8 steps) }\end{array}$ & $\begin{array}{l}\text { Danishefsky diene } \\
\text { (hetero-Diels-Alder) }\end{array}$ \\
\hline DeShong & $\begin{array}{l}\text { J. Am. Chem. Soc. } \\
\mathbf{1 9 8 4}, 106,5598\end{array}$ & $\begin{array}{l}\text { methyl daunosaminide } \\
\text { (racemic) }\end{array}$ & $\begin{array}{l}23 \% \\
\text { (7 steps) }\end{array}$ & $\begin{array}{l}\text { trans-crotonic acid } \\
\text { (nitrone cycloaddition) }\end{array}$ \\
\hline Hauser & $\begin{array}{l}\text { J. Org. Chem. } \\
\mathbf{1 9 8 4 , 4 9 , 2 2 3 6}\end{array}$ & $\begin{array}{l}N \text {-benzoyldaunosamine } \\
\text { (with classical resolution) }\end{array}$ & $\begin{array}{l}6.8 \% \\
\text { (9 steps) }\end{array}$ & $\begin{array}{l}\text { 1,3-pentadiene } \\
\text { (chlorosulfonyl isocyanate } \\
\text { cycloaddition }\end{array}$ \\
\hline Hauser & $\begin{array}{l}\text { J. Org. Chem. } \\
\mathbf{1 9 8 6}, 51,50\end{array}$ & $\begin{array}{l}N \text {-trichloroacetyldaunosamine } \\
\text { (racemic) }\end{array}$ & $\begin{array}{l}34 \% \\
(6 \text { steps })\end{array}$ & $\begin{array}{l}\text { 2,4-pentadien-1-ol } \\
\text { (imidate rearrangement) }\end{array}$ \\
\hline \multicolumn{5}{|c|}{ B. Chiral pool strategies ("chiron approach") } \\
\hline Parker & $\begin{array}{l}\text { Org. Lett. 2005, 7, } \\
1785\end{array}$ & $\begin{array}{l}\text { daunosamine glycal, } \\
\text { oxazolidinone derivative }\end{array}$ & $\begin{array}{l}30 \% \\
(7 \text { steps })\end{array}$ & $\begin{array}{l}\text { ethyl lactate } \\
\text { (allenylstannane addition, } \\
\text { Rh-cat nitrene insertion, W- } \\
\text { cat alkynol cyclization }\end{array}$ \\
\hline Sibi & $\begin{array}{l}\text { J. Org. Chem. } \\
\text { 1997, 62, } 5864\end{array}$ & $N$-Boc-daunosamine & $\begin{array}{l}15 \% \\
(7 \text { steps })\end{array}$ & $\begin{array}{l}\text { methyl lactate } \\
\text { (aldol, Curtius) }\end{array}$ \\
\hline \multicolumn{5}{|c|}{ C. Chiral reagent strategies } \\
\hline Davies & $\begin{array}{l}\text { Tetrahedron: } \\
\text { Asymmetry 1996, } \\
7,1273\end{array}$ & methyl daunosaminide & $\begin{array}{l}7.3 \% \\
\text { (5 steps) }\end{array}$ & $\begin{array}{l}\text { methyl sorbate } \\
\text { (asymmetric } 1,4 \text {-addition of } \\
\text { chiral amine) }\end{array}$ \\
\hline Sammes & $\begin{array}{l}\text { J. Chem. Soc. } \\
\text { Perkin Trans. } 1, \\
\mathbf{1 9 8 8 , ~} 111\end{array}$ & $\begin{array}{l}\text { methyl } N \text { - } \\
\text { benzoyldaunosaminide }\end{array}$ & $\begin{array}{l}33 \% \\
\text { (7 steps) }\end{array}$ & $\begin{array}{l}\text { 2-acetylfuran } \\
\text { (asymmetric reduction, } \\
\text { imidate rearrangement) }\end{array}$ \\
\hline \multicolumn{5}{|c|}{ D. Asymmetric catalysis strategies } \\
\hline Dai & $\begin{array}{l}\text { J. Am. Chem. Soc. } \\
\mathbf{1 9 8 8}, 110,5195\end{array}$ & $N$-benzoyldaunosamine & $\begin{array}{l}8.3 \% \\
(7 \text { steps }) \\
\end{array}$ & $\begin{array}{l}\text { 3-butyn-2-ol } \\
\text { (asymmetric epoxidation) }\end{array}$ \\
\hline Fuganti & $\begin{array}{l}\text { J. Org. Chem. } \\
\text { 1983, } 48,910\end{array}$ & $N$-benzoyldaunosamine & $\begin{array}{l}10 \% \\
\text { (6 steps) }\end{array}$ & $\begin{array}{l}\text { cinnamaldehyde } \\
\text { (enzymatic transformation, } \\
\text { allyl addition to chiral imine) }\end{array}$ \\
\hline Hoveyda & $\begin{array}{l}\text { Tetrahedron 1997, } \\
53,16377\end{array}$ & daunosamine $\bullet \mathrm{HCl}$ & $\begin{array}{l}10 \% \\
\text { (8 steps) }\end{array}$ & $\begin{array}{l}\text { ethyl sorbate } \\
\text { (asym. dihydroxylation, } \\
\text { nitrone cycloaddition) }\end{array}$ \\
\hline THIS WORK & & $\begin{array}{l}\text { methyl N-TFA- } \\
\text { daunosaminide }\end{array}$ & $\begin{array}{l}32 \% \\
\text { (9 steps) }\end{array}$ & $\begin{array}{l}\text { crotonaldehyde } \\
\text { (asym. dihydroxylation, } \\
\text { radical addition to imine) }\end{array}$ \\
\hline
\end{tabular}

